\title{
On the Construction of Approximate Solutions for the 1D Pollutant Transport Model
}

\author{
Yacouba $\mathrm{ZONGO}^{1}$, Brahima ROAMBA ${ }^{1,2}$ \& Boulaye YIRA $^{2}$ \\ ${ }^{1}$ UFR/ST, Université Nazi Boni, 01 BP 1091 Bobo-Dioulasso, Burkina Faso \\ 2 IUT, Université Nazi Boni, 01 BP 1091 Bobo-Dioulasso, Burkina Faso \\ Correspondence: Brahima ROAMBA, UFR/ST, Université Nazi Boni, 10 BP 1091 Bobo-Dioulasso, Burkina Faso
}

Received: March 10, 2020 Accepted: April 8, 2020 Online Published: April 10, 2020

doi:10.5539/jmr.v12n3p1 URL: https://doi.org/10.5539/jmr.v12n3p1

\begin{abstract}
The purpose of this paper is to build sequences of suitably smooth approximate solutions to the 1D pollutant transport model that preserve the mathematical structure discovered in (Roamba, Zabsonré, Zongo, 2017). The stability arguments in this paper then apply to such sequences of approximate solutions, which leads to the global existence of weak solutions for this model. We show that when the Reynold number goes to infinity, we have always an existence of global weak solutions result for the corresponding model.
\end{abstract}

Keywords: shallow water equations, bilayer models, viscosity, friction, capillarity, intermolecular forces, construction of weak solutions

\section{Introduction}

We consider a bilayer model of immiscible fluids where the upper layer can be represented by a Reynolds lubrifications model and the lower layer by a shallow water model. It can be used to simulate for instance the evolution of a pollutant fluid over water. A similar model was studied in (Fernandez-Nieto, Narbona-Reina \& Zabsonré, 2017). The model reads as follows:

$$
\begin{gathered}
\partial_{t} h_{1}+\partial_{x}\left(h_{1} u\right)=0, \\
\partial_{t}\left(h_{1} u\right)+\partial_{x}\left(h_{1} u^{2}\right)+\frac{1}{2} g \partial_{x} h_{1}^{2}-4 v_{1} \partial_{x}\left(h_{1} \partial_{x} u\right)+\frac{u}{\beta}-h_{1} \partial_{x}\left(\sigma \partial_{x}^{2} h_{1}-V\left(h_{1}\right)\right) \\
+r_{1} h_{1}|u|^{2} u+r g h_{1} \partial_{x} h_{2}+r g h_{2} \partial_{x}\left(h_{1}+h_{2}\right)=0, \\
\partial_{t} h_{2}+\partial_{x}\left(h_{2} u\right)-\varepsilon \partial_{x}^{2} h_{2}-\partial_{x}\left(\left(a h_{2}{ }^{2}+b h_{2}{ }^{3}\right) \partial_{x} p_{2}\right)=0,
\end{gathered}
$$

with

$$
\partial_{x} p_{2}=\rho_{2} g \partial_{x}\left(h_{1}+h_{2}\right) \quad \text { and } \quad V\left(h_{1}\right)=\frac{1}{h_{1}^{3}}-\frac{\alpha}{h_{1}^{4}} \quad(\alpha>0)
$$

where $(t, x) \in(0, T) \times] 0,1[$.

These equations represent a system composed of two layers of immiscible fluids.

Where we denote $h_{1}, h_{2}$ respectively, the water and the pollutant heights, $u$ is the water velocity. $v_{1}$ is the kinematic viscosity and $p_{2}$ the pressure; $g$ is the constant gravity. The coefficients $\sigma, r_{1}$ and $\beta$ are respectively the coefficients of the intrefaz tension, quadratic friction and positive slip length parameters; $a$ and $b$ respectively depend on the friction at the interfaz and coefficient of the viscosity of the pollutant. $\alpha, \varepsilon$ are positive constants. $r$ is the ratio of densities given by $r=\frac{\rho_{2}}{\rho_{1}}$ where $\rho_{1}$ and $\rho_{2}$ denoted respectively the densities of the water and the pollutant. $V\left(h_{1}\right)$ represents the force of Van Der Waals which is given by $V\left(h_{1}\right)=\frac{1}{h_{1}^{3}}-\frac{\alpha}{h_{1}^{4}}(\alpha>0)$, see (Kitavtsev, Laurençot \& Niethammer, 2011; Roamba, Zabsonré \& Zongo, 2017; Seemann, Herminghaus \& Jacobs, 2001).

We complete the system studied with the initial conditions

$$
h_{1}(0, x)=h_{1_{0}}(x), \quad h_{2}(0, x)=h_{2_{0}}(x), \quad\left(h_{1} u\right)(0, x)=\mathbf{m}_{0}(x) \quad \text { in }[0,1]
$$




$$
\begin{aligned}
& h_{1_{0}}, \quad h_{2_{0}} \in L^{2}(0,1), \quad \partial_{x}\left(h_{1_{0}}\right) \in L^{2}(0,1), \\
& \partial_{x} \mathbf{m}_{0} \in L^{1}(0,1), \quad \mathbf{m}_{0}=0 \quad \text { if } \quad h_{1_{0}}=0, \\
& \frac{\left|\mathbf{m}_{0}\right|^{2}}{h_{1_{0}}} \in L^{1}(0,1), \quad \varphi\left(h_{1_{0}}\right) \in L^{1}(0,1),
\end{aligned}
$$

where $\varphi\left(h_{1}\right)=4 v_{1} \log h_{1}$.

The energy inequality associated to the system (1)-(3) is:

$$
\begin{aligned}
& \frac{d}{d t} \int_{0}^{1}\left[\frac{1}{2} h_{1}|u|^{2}+U\left(h_{1}\right)+\frac{1}{2} g(1-r)\left|h_{1}\right|^{2}+\frac{1}{2} r g\left|h_{1}+h_{2}\right|^{2}+\frac{1}{2} \sigma\left|\partial_{x} h_{1}\right|^{2}\right] \\
& +4 v_{1} \int_{0}^{1} h_{1}\left|\partial_{x} u\right|^{2}+\frac{1}{\beta} \int_{0}^{1}|u|^{2}+\frac{1}{2} g r \varepsilon \int_{0}^{1}\left|\partial_{x} h_{2}\right|^{2} \\
& +r_{1} \int_{0}^{T} \int_{0}^{1} h_{1}|u|^{4}+\rho_{2} r g^{2} \int_{0}^{1} h_{2}^{2}\left|\partial_{x}\left(h_{1}+h_{2}\right)\right|^{2}\left(a+b h_{2}\right) \leq \frac{1}{2} r g \varepsilon \int_{0}^{1}\left|\partial_{x} h_{1}\right|^{2}
\end{aligned}
$$

where the potential function $U$ is the indefinite integral of $V$ defined by $U\left(h_{1}\right)=-\frac{1}{2 h_{1}^{2}}+\frac{\alpha}{3 h_{1}^{3}}, \quad h_{1}>0$. The entropy inequality associated with system (1)-(3) reads as

$$
\begin{gathered}
\quad \frac{d}{d t} \int_{0}^{1}\left[\frac{1}{2} h_{1}\left|u+\partial_{x} \varphi\left(h_{1}\right)\right|^{2}-\frac{1}{\beta} \varphi\left(h_{1}\right)+\frac{1}{2} g(1-r)\left|h_{1}\right|^{2}+\frac{1}{2} r g\left|h_{1}+h_{2}\right|^{2}+\frac{1}{2} \sigma\left|\partial_{x} h_{1}\right|^{2}+U\left(h_{1}\right)\right] \\
+\frac{1}{\beta} \int_{0}^{1}|u|^{2}+4 v_{1} \int_{0}^{1}\left(g+g r \frac{h_{2}}{h_{1}}+V^{\prime}\left(h_{1}\right)\right)\left|\partial_{x} h_{1}\right|^{2}+r g \int_{0}^{1}\left(\varepsilon+4 v_{1} \frac{h_{2}}{h_{1}}\right) \partial_{x} h_{1} \partial_{x} h_{2}+4 v_{1} \sigma \int_{0}^{1}\left|\partial_{x}^{2} h_{1}\right|^{2} \\
+r_{1} \int_{0}^{T} \int_{0}^{1} h_{1}|u|^{4}+g r \varepsilon \int_{0}^{1}\left|\partial_{x} h_{2}\right|^{2}+r g^{2} \int_{0}^{1} h_{2}^{2}\left(a+b h_{2}\right)\left(\partial_{x}\left(h_{1}+h_{2}\right)\right)^{2} \leq \frac{1}{2} r g \varepsilon \int_{0}^{1}\left|\partial_{x} h_{1}\right|^{2}
\end{gathered}
$$

We say that $\left(h_{1}, h_{2}, u\right)$ is a weak solution of (1)-(3), with the initial condition verifying the entropy inequality (8) for all smooth test functions $\phi=\phi(t, x)$ with $\phi(T,)=$.0 , we have:

$$
\begin{gathered}
h_{0_{1}} \phi(0, .)-\int_{0}^{T} \int_{0}^{1} h_{1} \partial_{t} \phi-\int_{0}^{T} \int_{0}^{1} h_{1} u \partial_{x} \phi=0 \\
-h_{0_{2}} \phi(0, .)-\int_{0}^{T} \int_{0}^{1} h_{2} \partial_{t} \phi-\int_{0}^{T} \int_{0}^{1} h_{2} u \partial_{x} \phi+\varepsilon \int_{0}^{T} \int_{0}^{1} \partial_{x} h_{2} \partial_{x} \phi \\
+\int_{0}^{T} \int_{0}^{1}\left(\left(a h_{2}{ }^{2}+b h_{2}{ }^{3}\right) \partial_{x} p_{2}\right) \partial_{x} \phi=0 \\
h_{01} u_{0} \phi(0, .)-\int_{0}^{T} \int_{0}^{1} h_{1} u \partial_{t} \phi-\int_{0}^{T} \int_{0}^{1} h_{1} u^{2} \partial_{x} \phi+4 v_{1} \int_{0}^{T} \int_{0}^{1} h_{1} \partial_{x} u \partial_{x} \phi \\
+\frac{1}{\beta} \int_{0}^{T} \int_{0}^{1} u \phi+\int_{0}^{T} \int_{0}^{1}\left(\sigma \partial_{x}^{2} h_{1}-V\left(h_{1}\right)\right) \phi \partial_{x} h_{1}+\int_{0}^{T} \int_{0}^{1}\left(\sigma \partial_{x}^{2} h_{1}-V\left(h_{1}\right)\right) h_{1} \partial_{x} \phi \\
-\frac{1}{2} g \int_{0}^{T} \int_{0}^{1} h_{1}^{2} \partial_{x} \phi-r g \int_{0}^{T} \int_{0}^{1} h_{2} h_{1} \partial_{x} \phi+r_{1} \int_{0}^{T} \int_{0}^{1} h_{1}|u|^{2} u \phi \\
-r g \int_{0}^{T} \int_{0}^{1} \phi h_{2} \partial_{x} h_{1}-r g \int_{0}^{T} \int_{0}^{1}\left(h_{1}+h_{2}\right) h_{2} \partial_{x} \phi-r g \int_{0}^{T} \int_{0}^{1}\left(h_{1}+h_{2}\right) \partial_{x} h_{2} \phi=0 .
\end{gathered}
$$

This work follows the work done in (Roamba, Zabsonré \& Zongo, 2017). In (Roamba, Zabsonré \& Zongo, 2017) as in this present work, we use a model of transport of pollutant in 1D formally derived in (Fernandez-Nieto, Narbona-Reina \& Zabsonré, 2013). In (Roamba, Zabsonré \& Zongo, 2017), the authors showed the existence of global weak solutions of similar model derived in (Fernandez-Nieto, Narbona-Reina \& Zabsonré, 2013). To lead well this result, the authors considered the condition according to which $h_{2} \leq h_{1}$ (the water layer is more important than the layer of the pollutant). We suppose in this paper the existence of molecular interactions between molecules and this leads us to use the Van Der 
Waals force which is given by $V\left(h_{1}\right)=\frac{1}{h_{1}^{3}}-\frac{\alpha}{h_{1}^{4}} \quad(\alpha>0)$, see (Kitavtsev, Laurençot \& Niethammer, 2011; Seemann, Herminghaus \& Jacobs, 2001). This force of Van Der Waals allows us to lower the height of water which allows us to get around hypothesis made in (Roamba, Zabsonré \& Zongo, 2017).

From a theoretical point of view several studies have been carried out on the construction of global weak solutions of shallow-water equations model. The construction of global weak solutions for a shallow water model is done in (Bresch \& Desjardins, 2006) for the two-dimensional case. In (Kitavtsev, Laurençot \& Niethammer, 2011), the authors, to prove the existence of global weak solutions for one-dimensional lubrification models, have constructed approximate solutions.

In this paper, our contribution is to build sequences of suitably smooth approximate solutions to the 1D pollutant transport model for a similar model studied in (Roamba, Zabsonré \& Zongo, 2017). A similar method of construction of weak solutions has been made in (Gamba, jüngel \& Vasseur, 2009). In (Vasseur \& Yu, 2016; Roamba \& Zabsonré, 2017), an other method of construction of weak solutions is developped to prove the existence of global weak solutions by deriving the Mellet-Vasseur type inequality.

We complete the system (1) - (3) by:

$$
\begin{gathered}
u=0 \quad \text { at } \quad x=0,1 \\
\partial_{x} h_{i}=0, \quad i=1,2 \quad \text { at } \quad x=0,1
\end{gathered}
$$

Our paper is organized as follows. On the one hand, the Section 2 is devoted to the actual construction of solutions to a perturbed system that preserves the BD entropy discovered in (Bresch \& Desjardins, 2002; Bresch \& Desjardins, 2006; Bresch, Desjardins \& Lin, 2003), we establish a classical energy equality and the "mathematical BD entropy", which entail some regularities on the unknowns. The BD entropy is a mathematical entropy introduced firstly in (Bresch \& Desjardins, 2002). Then we give a proposition allowing us to limit inferiorly the height of water which is very fundamental for the continuation since this limit study gives us additional regularities on the data. We also give an existence theorem of global weak solutions. To end, we give the proof of existence Theorem including the limits passage in the section.

\section{Construction of Approximate Solutions}

This section is devoted to the construction of approximate solutions to the 1D pollutant transport model. A small parameter $\beta$ is introduced. For given $\eta>0$, the approximate system is globally well posed and $h_{1}$ is bounded and bounded away from 0 . The global existence of weak solutions is obtained by taking the limit $\eta \rightarrow 0$ and using the stability arguments detailed in (Roamba, Zabsonré \& Zongo, 2017) and (Roamba, Zabsonré \& Zongo, 2017). Although the pressure term $V\left(h_{1}\right)$ does not need a regularization as in the case of Bresch and Desjardins (Bresch \& Desjardins, 2006), one still needs to regularize the function $h_{1}$ sufficiently in order to control additional higher order terms arising in the entropy equality. The approximating systems we take are given by

$$
\begin{gathered}
\partial_{t} h_{1_{\eta}}+\partial_{x}\left(h_{1_{\eta}} u_{\eta}\right)=0, \\
\partial_{t}\left(h_{1_{\eta}} u_{\eta}\right)+\partial_{x}\left(h_{1_{\eta}} u_{\eta}^{2}\right)+\frac{1}{2} g \partial_{x}\left(h_{1_{\eta}}\right)^{2}-4 v_{1} \partial_{x}\left(h_{1_{\eta}} \partial_{x} u_{\eta}\right)+\frac{u_{\eta}}{\beta}-h_{1_{\eta}} \partial_{x}\left(\sigma \partial_{x}^{2} h_{1_{\eta}}-V\left(h_{1_{\eta}}\right)\right) \\
+r g h_{1_{\eta}} \partial_{x} h_{2_{\eta}}+r g h_{2_{\eta}} \partial_{x}\left(h_{1_{\eta}}+h_{2_{\eta}}\right)-\eta h_{1_{\eta}}\left(\partial_{x}^{7} h_{1_{\eta}}+\partial_{x}^{3} h_{1_{\eta}}\right)+\eta^{2} \partial_{x}^{4} u_{\eta}=0, \\
\partial_{t} h_{2_{\eta}}+\partial_{x}\left(h_{2_{\eta}} u_{\eta}\right)-\eta\left(\partial_{x}^{6} h_{2_{\eta}}+\partial_{x}^{4} h_{2_{\eta}}\right)-\varepsilon \partial_{x}^{2} h_{2_{\eta}}-\partial_{x}\left(\left(a h_{2_{\eta}}{ }^{2}+b\left(h_{2_{\eta}}\right)^{3}\right) \partial_{x} p_{2_{\eta}}\right)=0,
\end{gathered}
$$

with

$$
\partial_{x} p_{2_{\eta}}=\rho_{2} g \partial_{x}\left(h_{1_{\eta}}+h_{2_{\eta}}\right) \quad \text { and } \quad V\left(h_{1_{\eta}}\right)=\frac{1}{\left(h_{1_{\eta}}\right)^{3}}-\frac{\alpha}{\left(h_{1_{\eta}}\right)^{4}} \quad(\alpha>0),
$$

where $(t, x) \in(0, T) \times] 0,1[$ and $\eta$ is a small parameter. Consider (14) - (16) with boundary condions

$$
u_{\eta}=\partial_{x}^{2} u_{\eta}=\partial_{x} h_{1_{\eta}}=\partial_{x}^{3} h_{1_{\eta}}=\partial_{x}^{5} h_{1_{\eta}}=\partial_{x} h_{2_{\eta}}=\partial_{x}^{5} h_{2_{\eta}}=0, \quad(t, x) \in(0, T) \times\{0,1\} .
$$

and initial data

$$
\begin{aligned}
& h_{1, \eta}^{0}, h_{2, \eta}^{0} \in H^{1}(0,1), \quad u_{\eta}^{0} \in L^{2}(0,1), \\
& u_{\eta}(x, 0)=u_{\eta}^{0}(x), \quad h_{1_{\eta}}(x, 0)=h_{1_{\eta}}^{0}(x)>0 \quad \text { and } \quad h_{2_{\eta}}(x, 0)=h_{2, \eta}^{0}(x)>0, \quad \text { in } \quad(0,1),
\end{aligned}
$$

where $u_{\eta}^{0}, h_{1_{\eta}}^{0}$ and $h_{2,0}^{\eta}$ are smooth functions such as

$$
\begin{aligned}
& u_{\eta}^{0} \rightarrow u_{1,0} \quad \text { in } L^{2}(0,1), \quad h_{1_{\eta}}^{0} \rightarrow h_{1,0}, \quad h_{2, \eta}^{0} \rightarrow h_{2,0} \quad \text { in } H^{1}(0,1) \\
& \text { and } \eta h_{1_{\eta}}^{0} \rightarrow 0, \quad \eta h_{2, \eta}^{0} \rightarrow 0 \quad \text { in } H^{3}(0,1) \quad \text { as } \eta \rightarrow 0 .
\end{aligned}
$$


We have the following energy inequality

Lemma 1. For classical solutions of the system (14)-(16), the following inequality holds

$$
\begin{aligned}
E\left(u_{\eta}, h_{1_{\eta}}, h_{2_{\eta}}\right)+ & 4 v_{1} \int_{0}^{T} \int_{0}^{1} h_{1_{\eta}}\left|\partial_{x} u_{\eta}\right|^{2}+\frac{1}{\beta} \int_{0}^{T} \int_{0}^{1}\left|u_{\eta}\right|^{2}+\frac{1}{2} r g \eta \int_{0}^{T} \int_{0}^{1}\left|\partial_{x}^{2} h_{2_{\eta}}\right|^{2} \\
+\frac{1}{2} g r \varepsilon & \int_{0}^{T} \int_{0}^{1}\left|\partial_{x} h_{2_{\eta}}\right|^{2}+r g^{2} \rho_{2} \int_{0}^{T} \int_{0}^{1}\left(h_{1_{\eta}}\right)^{2}\left(a+b h_{2_{\eta}}\right)\left(\partial_{x}\left(h_{1_{\eta}}+h_{2_{\eta}}\right)\right)^{2}+\eta^{2} \int_{0}^{T} \int_{0}^{1}\left|\partial_{x}^{2} u_{\eta}\right|^{2} \\
& +\frac{1}{2} r g \eta \int_{0}^{T} \int_{0}^{1}\left|\partial_{x}^{3} h_{2_{\eta}}\right|^{2} \leq \frac{1}{2} r g \int_{0}^{T} \int_{0}^{1}\left[\varepsilon\left|\partial_{x} h_{1_{\eta}}\right|^{2}+\eta\left|\partial_{x}^{2} h_{1_{\eta}}\right|^{2}+\eta\left|\partial_{x}^{3} h_{1_{\eta}}\right|^{2}\right]+E\left(u_{\eta}^{0}, h_{1_{\eta}}^{0}, h_{2,0}^{\eta}\right),
\end{aligned}
$$

where

$$
\begin{aligned}
& \begin{aligned}
E\left(u_{\eta}, h_{1_{\eta}}, h_{2_{\eta}}\right):=\int_{0}^{1} & {\left[\frac{1}{2} h_{1_{\eta}}\left|u_{\eta}\right|^{2}+U\left(h_{1_{\eta}}\right)+\frac{1}{2} g(1-r)\left|h_{1_{\eta}}\right|^{2}+\frac{1}{2} r g\left|h_{1_{\eta}}+h_{2_{\eta}}\right|^{2}+\frac{1}{2} \sigma\left|\partial_{x} h_{1_{\eta}}\right|^{2}+\eta \frac{1}{2}\left|\partial_{x}^{2} h_{1_{\eta}}\right|^{2}\right.} \\
& \left.+\frac{\eta}{2}\left|\partial_{x}^{3} h_{1_{\eta}}\right|^{2}\right],
\end{aligned} \\
& \text { and }
\end{aligned}
$$$$
U\left(h_{1}\right)=-\frac{1}{2 h_{1}^{2}}+\frac{\alpha}{3 h_{1}^{3}}, \quad h_{1}>0 .
$$

Remark 1. Notice that the two terms in the right can be controlled using Gronwall's lemma.

Remark 2. Let $\left(h_{1_{\eta}}, h_{2_{\eta}}, u_{\eta}\right)$ be a solution of model (14) - (16). Then, thanks to the energy inequality, we have:

$$
\begin{aligned}
& \sqrt{\frac{1}{2} g(1-r)} h_{1_{\eta}} \quad \text { is bounded in } \quad L^{\infty}\left(0, T ; L^{2}(0,1)\right) \text {, } \\
& \sqrt{\frac{1}{2} \sigma} \partial_{x} h_{1_{\eta}} \quad \text { is bounded in } \quad L^{\infty}\left(0, T ; L^{2}(0,1)\right), \\
& \sqrt{\frac{1}{2} r g}\left(h_{1_{\eta}}+h_{2_{\eta}}\right) \quad \text { is bounded in } \quad L^{\infty}\left(0, T ; L^{2}(0,1)\right) \text {, } \\
& \sqrt{\frac{1}{2}} \sqrt{h_{1_{\eta}}} u_{\eta} \quad \text { is bounded in } \quad L^{\infty}\left(0, T ; L^{2}(0,1)\right), \\
& 2 \sqrt{v_{1}} \sqrt{h_{1_{\eta}}} \partial_{x} u_{\eta} \quad \text { is bounded in } \quad L^{2}\left(0, T ; L^{2}(0,1)\right) \text {, } \\
& \frac{1}{\sqrt{\beta}} u_{\eta} \quad \text { is bounded in } \quad L^{2}\left(0, T ; L^{2}(0,1)\right), \\
& g \sqrt{r \rho_{2}} h_{2_{\eta}} \sqrt{a+b h_{2_{\eta}}}\left(\partial_{x}\left(h_{1_{\eta}}+h_{2_{\eta}}\right)\right) \quad \text { is bounded in } \quad L^{2}\left(0, T ; L^{2}(0,1)\right) \text {, } \\
& \sqrt{r g \eta}\left(h_{1_{\eta}}\right)^{-\frac{3}{2}} \quad \text { is bounded in } \quad L^{\infty}\left(0, T ; L^{2}(0,1)\right), \\
& \sqrt{\frac{\eta}{2}} \partial_{x}^{3} h_{1_{\eta}} \quad \text { is bounded in } \quad L^{\infty}\left(0, T ; L^{2}(0,1)\right), \\
& \sqrt{g r \varepsilon} \partial_{x} h_{2 \eta} \quad \text { is bounded in } \quad L^{2}\left(0, T ; L^{2}(0,1)\right) \text {, } \\
& \sqrt{\frac{\eta}{2}} \partial_{x}^{2} h_{1_{\eta}} \quad \text { is bounded in } \quad L^{\infty}\left(0, T ; L^{2}(0,1)\right), \\
& \eta\left|\partial_{x}^{2} u_{\eta}\right| \quad \text { is bounded in } \quad L^{2}\left(0, T ; L^{2}(0,1)\right) .
\end{aligned}
$$

The following lemma gives us the inequality of entropy necessary to limit $\partial_{x} \sqrt{h_{1_{\eta}}}$.

Lemma 2. For smooth solutions $\left(h_{1_{\eta}}, h_{2_{\eta}}, u_{\eta}\right)$ of model (14) - (16) satisfying the classical energy equality of the lemma 1 , we have the following mathematical BD entropy inequality:

$$
S\left(u_{\eta}, h_{1_{\eta}}, h_{2_{\eta}}\right)+\frac{1}{\beta} \int_{0}^{T} \int_{0}^{1}\left|u_{\eta}\right|^{2}+4 v_{1} \int_{0}^{T} \int_{0}^{1}\left(g+g r \frac{h_{2_{\eta}}}{h_{1_{\eta}}} V^{\prime}\left(h_{1_{\eta}}\right)\right)\left|\partial_{x} h_{1_{\eta}}\right|^{2}+4 r g v_{1} \int_{0}^{T} \int_{0}^{1}\left(1+\frac{h_{1_{\eta}}}{h_{1_{\eta}}}\right) \partial_{x} h_{1_{\eta}} \partial_{x} h_{2_{\eta}}
$$




$$
\begin{gathered}
+4 v_{1} \sigma \int_{0}^{T} \int_{0}^{1}\left|\partial_{x}^{2} h_{1_{\eta}}\right|^{2}+r g^{2} \rho_{2} \int_{0}^{T} \int_{0}^{1}\left(h_{1_{\eta}}\right)^{2}\left(a+b h_{2_{\eta}}\right)\left(\partial_{x}\left(h_{1_{\eta}}+h_{2_{\eta}}\right)\right)^{2}+\frac{1}{2} r g \eta \int_{0}^{T} \int_{0}^{1}\left|\partial_{x}^{2} h_{2_{\eta}}\right|^{2} \\
+\int_{0}^{T} \int_{0}^{1}\left[\eta^{2}\left|\partial_{x}^{2} u_{\eta}\right|^{2}+4 v \eta\left|\partial_{x}^{4} h_{1_{\eta}}\right|^{2}+4 v \eta^{2} \partial_{x}^{2} u_{\eta} \partial_{x}^{3} \log h_{1_{\eta}}\right] \\
\leq \frac{1}{2} r g \int_{0}^{T} \int_{0}^{1}\left[\varepsilon\left|\partial_{x} h_{1_{\eta}}\right|^{2}+\eta\left|\partial_{x}^{2} h_{1_{\eta}}\right|^{2}+\eta\left|\partial_{x}^{3} h_{1_{\eta}}\right|^{2}\right]+S\left(u_{\eta}^{0}, h_{1_{\eta}}^{0}, h_{2_{0}}^{\eta}\right),
\end{gathered}
$$

where

$$
\begin{aligned}
S\left(u_{\eta}, h_{1_{\eta}}, h_{2_{\eta}}\right):=\int_{0}^{1} & {\left[\frac{1}{2} h_{1_{\eta}}\left|u_{\eta}+\partial_{x} \varphi\left(h_{1_{\eta}}\right)\right|^{2}-\frac{1}{\beta} \varphi\left(h_{1_{\eta}}\right)+\frac{1}{2} r g\left|h_{1_{\eta}}+h_{2_{\eta}}\right|^{2}+\frac{1}{2} g(1-r)\left|h_{1_{\eta}}\right|^{2}\right.} \\
& \left.+\frac{1}{2} \sigma\left|\partial_{x} h_{1_{\eta}}\right|^{2}+U\left(h_{1_{\eta}}\right)+\frac{\eta}{2}\left|\partial_{x}^{2} h_{1_{\eta}}\right|^{2}+\frac{\eta}{2}\left|\partial_{x}^{3} h_{1_{\eta}}\right|^{2}\right] .
\end{aligned}
$$

\section{Remark 3.}

In the lemma 2 all the terms, excepted

$$
\int_{0}^{T} \int_{0}^{1}\left(\varepsilon+4 v_{1} \frac{h_{2_{\eta}}}{h_{1_{\eta}}}\right) \partial_{x} h_{1_{\eta}} \partial_{x} h_{2_{\eta}} \quad \int_{0}^{1} \int_{0}^{T} V^{\prime}\left(h_{1_{\eta}}\right)\left|\partial_{x} h_{1_{\eta}}\right|^{2} \quad \text { and } \quad \int_{0}^{T} \int_{0}^{1} 4 v \eta^{2} \partial_{x}^{2} u_{\eta} \partial_{x}^{3} \log h_{1_{\eta}}
$$

are controlled since they have the good sign. The control of the term $\int_{0}^{T} \int_{0}^{1}\left|u_{\eta}\right|^{2} u_{\eta} \partial_{x} h_{1_{\eta}}$ takes inspiration in (Roamba, Zabsonré \& Traor?, 2016). The term $\int_{0}^{1} \int_{0}^{T} V^{\prime}\left(h_{1_{\eta}}\right)\left|\partial_{x} h_{1_{\eta}}\right|^{2}$ can be absorbed thanks to the work done in (Kitavtsev, Laurençot \& Niethammer, 2011). It remains for us to control the terms

$\int_{0}^{T} \int_{0}^{1}\left(\varepsilon+4 v_{1} \frac{h_{2_{\eta}}}{h_{1_{\eta}}}\right) \partial_{x} h_{1_{\eta}} \partial_{x} h_{2_{\eta}}, \quad \int_{0}^{T} \int_{0}^{1} 4 v \eta^{2} \partial_{x}^{2} u_{\eta} \partial_{x}^{3} \log h_{1_{\eta}}$, see (Roamba, Zabsonré \& Zongo, 2017) and (Kitavtsev, Laurençot \& Niethammer, 2011) for justifications.

Remark 4. Given $\eta>0$, the equation (14) is parabolic in $u_{\eta}$. Also, the quations (15) and (16) are parabolic respectively in $h_{1_{\eta}}$ and in $h_{2_{\eta}}$. Relying on the works of Bresch and Desjardins in (Bresch \& Desjardins, 2006) and those of Kitavtsev, Laurençot and Niethammer in (Kitavtsev, Laurençot \& Niethammer, 2011) the system (14)-(16) with (19)-(20) has a unique classical solution at least locally in time. Arguing as in (Kitavtsev, Laurençot \& Niethammer, 2011), the Proposition 1 and the regularities above guarantee the global in time solvability for (14)-(16) with (19)-(20).

Proposition 1. If $h_{1_{\eta}}$ has the regularities established in corollary 2.1, then there exists constants $c_{1}$ and $c_{2}$ such as $0<c_{1}<h_{1_{\eta}}<c_{2}$.

Lemma 3. For classical solutions of the system (14) - (16) with a first component $h_{1_{\eta}}$, we have

$$
\frac{1}{4} \int_{0}^{1} h_{1_{\eta}}\left|\partial_{x} \varphi\left(h_{1_{\eta}}\right)\right|^{2} \leq \frac{1}{2} \int_{0}^{1} h_{1_{\eta}}\left(u_{\eta}+\partial_{x} \varphi\left(h_{1_{\eta}}\right)\right)^{2}+2 E\left(h_{1_{\eta}}, h_{2_{\eta}}, u_{\eta}\right)+\frac{1}{3 \alpha^{2}}
$$

with

$$
E\left(h_{1_{\eta}}, h_{2_{\eta}}, u_{\eta}\right)=\int_{0}^{1}\left[\frac{1}{2} h_{1_{\eta}}\left|u_{\eta}\right|^{2}+U\left(h_{1_{\eta}}\right)+\frac{1}{2} g(1-r)\left|h_{1_{\eta}}\right|^{2}+\frac{1}{2} r g\left|h_{1_{\eta}}+h_{2_{\eta}}\right|^{2}+\frac{1}{2} \sigma\left|\partial_{x} h_{1_{\eta}}\right|^{2}+\frac{\eta}{2}\left|\partial_{x}^{3} h_{1_{\eta}}\right|^{2}\right] .
$$

Proof: See (Roamba, Zabsonré \& Zongo, 2017).

Corollary 1. Let $\left(h_{1_{\eta}}, h_{2_{\eta}}, u_{\eta}\right)$ be a solution of model (14) - (16).

Then, thanks to lemma 3 and the BD entropy equality, we have:

$$
\begin{array}{cc}
\sqrt{h_{1_{\eta}}} \quad \text { is bounded in } & L^{\infty}\left(0, T ; L^{2}(0,1)\right), \\
\partial_{x} \sqrt{h_{1_{\eta}}} \quad \text { is bounded in } & L^{\infty}\left(0, T ; L^{2}(0,1)\right), \\
\partial_{x}^{2} h_{1_{\eta}} \quad \text { is bounded in } & L^{2}\left(0, T ; L^{2}(0,1)\right), \\
\sqrt{\eta} \partial_{x}^{2} h_{2_{\eta}} \quad \text { is bounded in } & L^{2}\left(0, T ; L^{2}(0,1)\right), \\
2 \sqrt{\eta} \partial_{x}^{4} h_{1_{\eta}} \quad \text { is bounded in } & L^{2}\left(0, T ; L^{2}(0,1)\right) .
\end{array}
$$


Remark 5. 1. In the remark 2, the estimate

$$
\sqrt{h_{1_{\eta}}} u_{\eta} \quad \text { is bounded in } \quad L^{\infty}\left(0, T ; L^{2}(0,1)\right)
$$

implies,

this leads us

$$
h_{1_{\eta}} u_{\eta} \quad \text { is bounded in } \quad L^{\infty}\left(0, T ; L^{2}(0,1)\right)
$$

$$
\partial_{t} h_{1_{\eta}} \quad \text { is bounded in } \quad L^{\infty}\left(0, T ; W^{-1,2}(0,1)\right) .
$$

2. We have the additional regularities thanks to Corollary 1:

(a) $h_{1_{\eta}}, u_{\eta}$ are bounded in $L^{2}\left(0, T ; H^{2}(0,1)\right)$,

(b) $h_{2_{\eta}}$ are bounded in $L^{2}\left(0, T ; H^{2}(0,1)\right)$,

(c) $h_{1_{\eta}} u_{\eta}$ is bounded in $L^{3}\left(0, T ; L^{3}(0,1)\right) \cap L^{\infty}\left(0, T ; L^{2}(0,1)\right)$.

Remark 6. We have the following additional regularities:

1. $h_{1_{\eta}}$ and $h_{2_{\eta}}$ are bounded in $L^{\infty}\left(0, T ; L^{2}(0,1)\right)$.

2. $\sqrt{h_{1_{\eta}}}$ is bounded in $L^{2}\left(0, T ; H^{1}(0,1)\right)$.

Indeed,

by integrating the mass equation, we obtain directly $\sqrt{h_{1_{\eta}}}$ in $L^{\infty}\left(0, T ; L^{2}(\Omega)\right)$. As Corollary 1 gives us $\partial_{x} \sqrt{h_{1_{\eta}}}$ in $L^{\infty}\left(0, T ; L^{2}(\Omega)\right)$, so $\sqrt{h_{1_{\eta}}}$ is bounded in $L^{\infty}\left(0, T ; H^{1}(\Omega)\right)$.

Remark 7. Since we have $0<c_{1}<h_{1_{\eta}}<c_{2}$ uniformly with respect to $\varepsilon$, the limit $h_{1_{\eta}}$ is bounded and bounded away from zero. The limit system can then be divided by $h_{1_{\eta}}$ and becomes parabolic with respect to the velocity $u_{\eta}$. Arguing as in Bresch and Desjardins (Bresch \& Desjardins, 2006), Kitavtsev, Laurençot and Niethammer (Kitavtsev, Laurençot \& Niethammer, 2011) the initial-boundary value problem system (14)-(16) with (18)-(19) has a unique classical solution at least locally in time.

Now, we are going to define a weak formulation of the problem (14)-(16) with boundary conditions (18). Consider $\left(h_{1_{0}}, h_{2_{0}}, u_{0}\right)$ satisfying $(20)$.

Definition 1. A triplet $\left(h_{1_{\eta}}, h_{2_{\eta}}, u_{\eta}\right)$ is a global weak solution to (14)-(16) with boundary conditions (18) and initial conditions $\left(h_{1_{0}}, h_{2_{0}}, u_{1_{0}}\right)$ if $h_{1_{0}}, h_{2_{0}}$ and $u_{0}$ enjoy the regularity properties stated above in this section and the following holds

$$
\begin{gathered}
h_{1_{\eta}}^{0} \phi(0, .)-\int_{0}^{T} \int_{0}^{1} h_{1_{\eta}} \partial_{t} \phi-\int_{0}^{T} \int_{0}^{1} h_{1_{\eta}} u_{\eta} \partial_{x} \phi=0 \\
-h_{2_{0}}^{\eta} \phi(0, .)-\int_{0}^{T} \int_{0}^{1} h_{2_{\eta}} \partial_{t} \phi-\int_{0}^{T} \int_{0}^{1} h_{2_{\eta}} u_{\eta} \partial_{x} \phi+\varepsilon \int_{0}^{T} \int_{0}^{1} \partial_{x} h_{2_{\eta}} \partial_{x} \phi \\
+\int_{0}^{T} \int_{0}^{1}\left(\left(a h_{2_{\eta}}{ }^{2}+b h_{2_{\eta}}{ }^{3}\right) \partial_{x} p_{2_{\eta}}\right) \partial_{x} \phi-\eta \int_{0}^{1} \int_{0}^{T} \partial_{x}^{6} h_{2_{\eta}} \phi-\eta \int_{0}^{1} \int_{0}^{T} \partial_{x}^{4} h_{2_{\eta}} \phi=0 \\
h_{0_{1}}^{\eta} u_{\eta}^{0} \phi(0, .)-\int_{0}^{T} \int_{0}^{1} h_{1_{\eta}} u_{\eta} \partial_{t} \phi-\int_{0}^{T} \int_{0}^{1} h_{1_{\eta}} u_{\eta}{ }^{2} \partial_{x} \phi+4 v_{1} \int_{0}^{T} \int_{0}^{1} h_{1_{\eta}} \partial_{x} u_{\eta} \partial_{x} \phi+\frac{1}{\beta} \int_{0}^{T} \int_{0}^{1} u_{\eta} \phi \\
+\int_{0}^{T} \int_{0}^{1}\left(\sigma \partial_{x}^{2} h_{1_{\eta}}-V\left(h_{1_{\eta}}\right)\right) \phi \partial_{x} h_{1_{\eta}}+\int_{0}^{T} \int_{0}^{1}\left(\sigma \partial_{x}^{2} h_{1_{\eta}}-V\left(h_{1_{\eta}}\right)\right) h_{1_{\eta}} \partial_{x} \phi-\frac{1}{2} g \int_{0}^{T} \int_{0}^{1}\left(h_{1_{\eta}}\right)^{2} \partial_{x} \phi \\
-r g \int_{0}^{T} \int_{0}^{1} h_{2_{\eta}} h_{1_{\eta}} \partial_{x} \phi+r_{1} \int_{0}^{T} \int_{0}^{1} h_{1_{\eta}}\left|u_{\eta}\right|^{2} u_{\eta} \phi-r g \int_{0}^{T} \int_{0}^{1} \phi h_{2_{\eta}} \partial_{x} h_{1_{\eta}}-\eta \int_{0}^{1} \int_{0}^{T} \partial_{x}^{2} u_{\eta} \partial_{x}^{2} \phi \\
-r g \int_{0}^{T} \int_{0}^{1}\left(h_{1_{\eta}}+h_{2_{\eta}}\right) h_{2_{\eta}} \partial_{x} \phi-r g \int_{0}^{T} \int_{0}^{1}\left(h_{1_{\eta}}+h_{2_{\eta}}\right) \partial_{x} h_{2_{\eta}} \phi+\eta \int_{0}^{1} \int_{0}^{T} h_{1_{\eta}} \partial_{x}^{7} h_{1_{\eta}} \phi-\eta \int_{0}^{1} \int_{0}^{T} \partial_{x}^{3} h_{1_{\eta}} \phi=0
\end{gathered}
$$

for all $\phi \in C_{0}^{\infty}([0, \infty) \times[0,1])$ such that $\phi(T,)=$.0 .

We now show that solutions to the system (14)-(16) with boundary and initial conditions (18)-(19) converge to a solution of (9)-(11) as $\eta \longrightarrow 0$.

Theorem 1. For any positive $\sigma, \beta$ and initial data $\left(h_{1_{0}}, h_{2_{0}}, u_{0}\right)$ satisfaying $(20)$, there exists a global weak solution to the system (14)-(16) with boundary conditions (18) and initial conditions (19) in the sense of (24)-(26). 


\section{Case $\beta=\infty$}

We follow the ideas proposed in (Kitavtsev, Laurençot \& Niethammer, 2011).

Let us first consider a sequence of positive real numbers $\left(\beta_{n}\right), \beta_{n} \rightarrow \infty$, and denote the corresponding solutions to (24)-(26) with $\beta=\beta_{n}$ by $\left(h_{1_{\beta_{n}}}, h_{2_{\beta_{n}}}, u_{\beta_{n}}\right)$. The corresponding system reads as:

$$
\begin{gathered}
\partial_{t} h_{1_{\beta_{n}}}+\partial_{x}\left(h_{1_{\beta n}} u_{\beta_{n}}\right)=0, \\
\partial_{t}\left(h_{1_{\beta_{n}}} u_{\beta_{n}}\right)+\partial_{x}\left(h_{1_{\beta_{n}}} u_{\beta_{n}}{ }^{2}\right)+\frac{1}{2} g \partial_{x}\left(h_{1_{\beta_{n}}}\right)^{2}-4 v_{1} \partial_{x}\left(1_{\beta_{n}} \partial_{x} u_{\beta_{n}}\right)+\frac{u_{\beta_{n}}}{\beta_{n}}-h_{1_{\eta}} \partial_{x}\left(\sigma \partial_{x}^{2} h_{1_{\beta_{n}}}-V\left(h_{1_{\beta_{n}}}\right)\right) \\
+r g h_{1_{\beta_{n}}} \partial_{x} h_{2_{\beta_{n}}}+r g h_{2_{\beta_{n}}} \partial_{x}\left(h_{1_{\beta_{n}}}+h_{2_{\beta_{n}}}\right)-\eta_{n} h_{1_{\beta_{n}}}\left(\partial_{x}^{7} h_{1_{\beta_{n}}}+\partial_{x}^{3} h_{1_{\beta_{n}}}\right)+\eta_{n}^{2} \partial_{x}^{4} u_{\beta_{n}}=0, \\
\partial_{t} h_{2_{\beta_{n}}}+\partial_{x}\left(h_{2_{\beta_{n}}} u_{\beta_{n}}\right)-\eta_{n}\left(\partial_{x}^{6} h_{2_{\beta_{n}}}+\partial_{x}^{4} h_{2_{\beta_{n}}}\right)-\varepsilon \partial_{x}^{2} h_{2_{\beta_{n}}}-\partial_{x}\left(\left(a h_{{2_{\beta}}}{ }^{2}+b\left(h_{2_{\beta_{n}}}\right)^{3}\right) \partial_{x} p_{2_{\beta_{n}}}\right)=0,
\end{gathered}
$$

with

$$
\partial_{x} p_{1_{\beta_{n}}}=\rho_{2} g \partial_{x}\left(h_{1_{\beta_{n}}}+h_{2_{\beta_{n}}}\right) \quad \text { and } \quad V\left(h_{1_{\beta_{n}}}\right)=\frac{1}{\left(h_{1_{\beta_{n}}}\right)^{3}}-\frac{\alpha}{\left(h_{1_{\beta_{n}}}\right)^{4}} \quad(\alpha>0),
$$

where $(t, x) \in(0, T) \times] 0,1\left[\right.$ and $\eta_{n}$ is a small parameter.

For this system (27) - (29), the statement of Remark 2, Corollary 1 and the Lemma 1 are true for the weak solutions to (24)-(26). We may then investigate the behaviour of these solutions as either $\beta \rightarrow \infty$. Though the estimate on $\left(u_{\beta_{n}} / \beta_{n}\right)$ is useless in that case, one still recovers the estimate of $\left(u_{\beta_{n}}\right)$ in $L^{2}\left(0, T ; H_{0}^{1}(0,1)\right)$ as a consequence of Remark 2 , Corollary 1 and the Poincaré inequality. Arguing as in the proof of Theorem 1, we conclude that, after possibly extracting a subsequence, $\left(h_{1_{\beta n}}, h_{2_{\beta n}}, u_{\beta_{n}}\right)$ converges towards a weak solution to the model

$$
\begin{gathered}
\partial_{t} h_{1}+\partial_{x}\left(h_{1} u\right)=0 \\
\partial_{t}\left(h_{1} u\right)+\partial_{x}\left(h_{1} u^{2}\right)+\frac{1}{2} g \partial_{x} h_{1}^{2}-4 v_{1} \partial_{x}\left(h_{1} \partial_{x} u\right)-h_{1} \partial_{x}\left(\sigma \partial_{x}^{2} h_{1}-V\left(h_{1}\right)\right) \\
+r_{1} h_{1}|u|^{2} u+r g h_{1} \partial_{x} h_{2}+r g h_{2} \partial_{x}\left(h_{1}+h_{2}\right)=0 \\
\partial_{t} h_{2}+\partial_{x}\left(h_{2} u\right)-\varepsilon \partial_{x}^{2} h_{2}-\partial_{x}\left(\left(a h_{2}{ }^{2}+b h_{2}{ }^{3}\right) \partial_{x} p_{2}\right)=0
\end{gathered}
$$

\section{Conclusion}

This article was the subject of the construction of global weak solutions of a model of pollutant transport in dimension 1. Furthermore, we have shown that the existence of global weak solutions of the model is preserved when the Reynolds number tends to infinity. For our future works, we will show the existence of global weak solutions of the model studied in this paper when $\sigma \longrightarrow 0$.

\section{Acknowledgments}

The authors thank Pr Jean De Dieu ZABSONRE for initiating this work and for his valuable comments on the paper.

\section{References}

Bresch, D., \& Desjardins, B. (2003). Existence of global weak solution for 2D viscous shallow water equations and convergence to the quasi-geostrophic model. Comm. Math. Phys., 238(1-3), 211-223. https://doi.org/10.1007/s00220-003-0859-8

Bresch, D., \& Desjardins, B. (2006). On the construction of approximate solutions for the 2D viscous shallow water model and for compressible Navier-Stokes models. J. Math. Pures Appl., 86, 362-368. https://doi:10.1016/j.matpur.2006.06.005

Bresch, D., \& Desjardins. (2007). On the existence of global weak solutions to the Navier-Stokes equations for viscous compressible and heat conducting fluids. J. Math. Pures Appl., 86(4), 362-368. https://doi.org/10.1081/PDE120020499

Bresch, D., Desjardins, B., \& Lin, C. K. (2003). On some compressible fluid models: Korteweg, lubrication and shallow water systems. Communications in partial differential equations, 28(3,4), 843-868.

https://doi.org/10.1081/PDE-120020499 
Fernandez-Nieto, E. D., Narbona-Reina, G., \& Zabsonré, J. D. (2013). Formal derivation of a bilayer model coupling shallow water and Reynolds lubrication equations: evolution of a thin pollutant layer over water. European Journal of Applied Mathematics, 24(6), 803-833. https://doi.org/10.1017/S095679251300020X

Gamba, I., Jüngel, G., \& Vasseur, A. (2009). Global existence of solutions to one-dimensional viscous quantum hydrodynamic equations. Jrl. of Diff. Equations, 247(11), 3117-3135. https://doi.org/10.1016/j.jde.2009.09.001

Kitavtsev, G., Laurençot, P., \& Niethammer, B. (2011). Weak solutions to lubrication equations in the presence of strong slippage. Methods and Applications of Analysis, 18, 183-202.

Lions, J. L. (1969). Quelques Methodes de Resolution des Probl?mes aux Limites Non Linéaires, Dunod.

Roamba, B., \& Zabsonré, J. D. D. (2017). On a bidimensional bi-layer shallow-water model. Electron. J. Differential Equation, (168), 1-19.

Roamba, B., Zabsonré, J. D. D., \& Traoré, S. (2016). Formal derivation and existence of global weak solutions of a two-dimensional bilayer model coupling shallow water and Reynolds lubrication equations. Asymptotic Analysis, 99, 207-239. https://doi.org/10.3233/ASY-16138

Roamba, B., Zabsonré, J. D. D., \& Zongo, Y. (2017). Weak solutions to pollutant transport model in a dimensional. Annals of the University of Craiova,Mathematics and Computer Science Series, 44(1), 137-148.

Roamba, B., Zabsonré, J. D. D., \& Zongo, Y. (2017). On the existence of global weak solutions to 1D pollutant transport model. Journal of Mathematics Research, 9(4). http://dx.doi.org/10.5539/jmr.v9n4p124

Seemann, R., Herminghaus, S., \& Jacobs, K. (2001). Dewetting Patterns and Molecular Forces: A Reconciliation. PHYSICAL REVIEW LETTERS, 86(24), 5534-5537. https://doi.org/10.1103/PhysRevLett.86.5534

Vasseur, A., \& Yu, C. (2016). Existence of global weak solution for 3D degenerate compressible Navier-Stokes equations. Inv. Math., 206(2), 935-974. https://doi.org/10.1007/s00222-016-0666-4

Simon, J. (1987). Compact set in the space $L^{p}(0 ; t ; B)$. Ann. Mat. Pura Appl., 146(4), 65-96. https://doi.org/10.1007/BF01762360

\section{Appendix}

Tha aim of this appendix is to prove technical lemmas that will yield crucial estimates for passing to the limits in the approximate (14)-(16).

\section{Proof of Lemma 1}

First, we multiply the momentum equation by $u_{\eta}$ and we integrate from 0 to 1 . We use the mass conservation equation of the first layer for simplification. Then, we obtain

$$
\begin{gathered}
\int_{0}^{1} \frac{1}{2} \partial_{t}\left(h_{1_{\eta}}\left(u_{\eta}\right)^{2}\right)+\frac{1}{2} \int_{0}^{1} g \partial_{x}\left(h_{1_{\eta}}\right)^{2} u_{\eta}-4 \int_{0}^{1} \partial_{x}\left(v_{1} h_{1_{\eta}} \partial_{x} u_{\eta}\right) u_{\eta}-\int_{0}^{1} h_{1_{\eta}} u_{\eta} \partial_{x}\left(\sigma \partial_{x}^{2} h_{1_{\eta}}-V\left(h_{1_{\eta}}\right)\right) \\
+\int_{0}^{1} \frac{\left(u_{\eta}\right)^{2}}{\beta}+\eta \int_{0}^{1} h_{1_{\eta}} \partial_{x}^{7} h_{1_{\eta}} u_{\eta}-\eta^{2} \int_{0}^{1} \partial_{x}^{4} u_{\eta} u_{\eta}+r g \int_{0}^{1} h_{1_{\eta}} \partial_{x} h_{2_{\eta}} u_{\eta} \\
+r g \int_{0}^{1} h_{2_{\eta}} \partial_{x}\left(h_{1_{\eta}}+h_{2_{\eta}}\right) u_{\eta}=0 .
\end{gathered}
$$

Now, we simplify each term as follows:

$$
\begin{aligned}
&-4 \int_{0}^{1} \partial_{x}\left(v_{1} h_{1_{\eta}} \partial_{x} u_{\eta}\right) u_{\eta}=4 v_{1} \int_{0}^{1} h_{1_{\eta}}\left(\partial_{x} u_{\eta}\right)^{2}, \\
&--\int_{0}^{1} h_{1_{\eta}} u_{\eta} \partial_{x}\left(\sigma \partial_{x}^{2} h_{1_{\eta}}-V\left(h_{1_{\eta}}\right)\right)=\int_{0}^{1} \partial_{x}\left(h_{1_{\eta}} u_{\eta}\right)\left(\sigma \partial_{x}^{2} h_{1_{\eta}}-V\left(h_{1_{\eta}}\right)\right) \\
&=-\int_{0}^{1} \partial_{t} h_{1_{\eta}}\left(\sigma \partial_{x}^{2} h_{1_{\eta}}-V\left(h_{1_{\eta}}\right)\right) \\
&=\int_{0}^{1} \sigma \partial_{x t} h_{1_{\eta}} \partial_{x} h_{1_{\eta}}+\int_{0}^{1} \partial_{t}\left(U\left(h_{1_{\eta}}\right)\right) \\
&=\int_{0}^{1} \partial_{t}\left(\frac{1}{2} \sigma\left|\partial_{x} h_{1_{\eta}}\right|^{2}+U\left(h_{1_{\eta}}\right)\right),
\end{aligned}
$$


- $r g \int_{0}^{1} h_{1_{\eta}} \partial_{x} h_{2_{\eta}} u_{\eta}=-r g \int_{0}^{1} h_{2_{\eta}} \partial_{x}\left(h_{1_{\eta}} u_{\eta}\right)=r g \int_{0}^{1} h_{2_{\eta}} \partial_{t} h_{1_{\eta}}$,

- $\frac{1}{2} g \int_{0}^{1} \partial_{x}\left(h_{1_{\eta}}\right)^{2} u_{\eta}=\frac{1}{2} g \frac{d}{d t} \int_{0}^{1}\left|h_{1_{\eta}}\right|^{2}$,

$\bullet \eta \int_{0}^{1} h_{1_{\eta}} \partial_{x}^{7} h_{1_{\eta}} u_{\eta}=-\eta \int_{0}^{1} \partial_{x}\left(h_{1_{\eta}} u_{\eta}\right) \partial_{x}^{6} h_{1_{\eta}}$

$$
\begin{aligned}
& =\eta \int_{0}^{1} \partial_{t} h_{1_{\eta}} \partial_{x}^{6} h_{1_{\eta}} \\
& =-\eta \int_{0}^{1} \partial_{x t} h_{1_{\eta}} \partial_{x}^{5} h_{1_{\eta}}
\end{aligned}
$$$$
=\eta \int_{0}^{1} \partial_{t} \partial_{x}^{2} h_{1_{\eta}} \partial_{x}^{4} h_{1_{\eta}}
$$$$
=-\eta \int_{0}^{1} \partial_{t} \partial_{x}^{3} h_{1_{\eta}} \partial_{x}^{3} h_{1_{\eta}}
$$

$$
=\frac{-\eta}{2} \frac{d}{d t} \int_{0}^{1}\left|\partial_{x}^{3} h_{1_{\eta}}\right|^{2}
$$

$\bullet \eta \int_{0}^{1} h_{1_{\eta}} \partial_{x}^{3} h_{1_{\eta}} u_{\eta}=-\eta \int_{0}^{1} \partial_{x}\left(h_{1_{\eta}} u_{\eta}\right) \partial_{x}^{2} h_{1_{\eta}}$

$$
\begin{aligned}
& =\eta \int_{0}^{1} \partial_{t} h_{1_{\eta}} \partial_{x}^{2} h_{1_{\eta}} \\
& =-\eta \int_{0}^{1} \partial_{x t} h_{1_{\eta}} \partial_{x} h_{1_{\eta}} \\
& =-\eta \frac{1}{2} \frac{d}{d t} \int_{0}^{1}\left|\partial_{x} h_{1_{\eta}}\right|^{2},
\end{aligned}
$$

$\bullet \eta^{2} \int_{0}^{1} u_{\eta} \partial_{x}^{4} u_{\eta}=-\eta^{2} \int_{0}^{1} \partial_{x} u_{\eta} \partial_{x}^{3} u_{\eta}$

$$
=\eta^{2} \int_{0}^{1}\left|\partial_{x}^{2} u_{\eta}\right|^{2}
$$

- $r g \int_{0}^{1} h_{2_{\eta}} \partial_{x}\left(h_{1_{\eta}}+h_{1_{\eta}}\right) u_{\eta}=-r g \int_{0}^{1}\left(h_{1_{\eta}}+h_{2_{\eta}}\right) \partial_{x}\left(h_{2_{\eta}} u_{\eta}\right)$.

The pollutant transport equation gives us:

$\partial_{x}\left(h_{2_{\eta}} u_{\eta}\right)=-\partial_{t} h_{2_{\eta}}+\varepsilon \partial_{x}^{2} h_{2_{\eta}}+\partial_{x}\left(\left(a h_{1_{\eta}}{ }^{2}+b h_{2_{\eta}}{ }^{3}\right) \partial_{x} p_{2_{\eta}}\right)+\eta\left(\partial_{x}^{6} h_{2_{\eta}}+\partial_{x}^{4} h_{2_{\eta}}\right)$ and we have:

- $r g \int_{0}^{1} h_{2_{\eta}} \partial_{x}\left(h_{1_{\eta}}+h_{1_{\eta}}\right) u_{\eta}=r g \varepsilon \int_{0}^{1} \partial_{x} h_{1_{\eta}} \partial_{x} h_{2_{\eta}}+r g \varepsilon \int_{0}^{1}\left|\partial_{x} h_{2_{\eta}}\right|^{2}+\frac{1}{2} r g \frac{d}{d t} \int_{0}^{1}\left|h_{2_{\eta}}\right|^{2}$

$$
\begin{aligned}
& +\rho_{2} r g^{2} \int_{0}^{1}\left(h_{1_{\eta}}\right)^{2}\left|\partial_{x}\left(h_{1_{\eta}}+h_{1_{\eta}}\right)\right|^{2}\left(a+b h_{2_{\eta}}\right)+r g \int_{0}^{1} h_{1_{\eta}} \partial_{t} h_{2_{\eta}}+r g \eta \int_{0}^{1} \partial_{x}^{3} h_{1_{\eta}} \partial_{x}^{3} h_{2_{\eta}} \\
& +r g \eta \int_{0}^{1}\left|\partial_{x}^{3} h_{2_{\eta}}\right|^{2}+r g \eta \int_{0}^{1} \partial_{x}^{2} h_{1_{\eta}} \partial_{x}^{2} h_{2_{\eta}}+r g \eta \int_{0}^{1}\left|\partial_{x}^{2} h_{2_{\eta}}\right|^{2} .
\end{aligned}
$$

Substituting all these terms in (34), we get (21) by integrating under 0 to $T$.

\section{Proof of Lemma 2}

Let us multiply the equation (15) by $\partial_{x} \varphi\left(h_{1_{\eta_{k}}}\right)$, integrate with respect to $x$ and use an integration by parts, and using (1), we have:

$4 v_{1} \int_{0}^{1}\left(\partial_{t} u_{\eta}+u_{\eta} \partial_{x} u_{\eta}\right) \partial_{x} h_{1_{\eta}}+4 v_{1} g \int_{0}^{1}\left|\partial_{x} h_{1_{\eta}}\right|^{2}+16 v_{1}^{2} \int_{0}^{1} h_{1_{\eta}} \partial_{x} u_{\eta} \partial_{x}\left(\frac{\partial_{x} h_{1_{\eta}}}{h_{1_{\eta}}}\right)$ 


$$
\begin{aligned}
+4 v_{1} \int_{0}^{1} \frac{u_{\eta} \partial_{x} h_{1_{\eta}}}{\beta h_{1_{\eta}}}+4 v_{1} \sigma \int_{0}^{1}\left|\partial_{x}^{2} h_{1_{\eta}}\right|^{2}+4 v_{1} \int_{0}^{1} V^{\prime}\left(h_{1_{\eta}}\right)\left|\partial_{x} h_{1_{\eta}}\right|^{2}+4 v_{1} r g \int_{0}^{1} \partial_{x} h_{1_{\eta}} \partial_{x} h_{1_{\eta}} \\
+4 v_{1} r g \int_{0}^{1} \frac{h_{2_{\eta}}}{h_{1_{\eta}}}\left|\partial_{x} h_{1_{\eta}}\right|^{2}+4 v_{1} r g \int_{0}^{1} \frac{h_{2_{\eta}}}{h_{1_{\eta}}} \partial_{x} h_{2_{\eta}} \partial_{x} h_{1_{\eta}}+4 v_{1} \eta \int_{0}^{1}\left|\partial_{x}^{4} h_{1_{\eta}}\right|^{2}+\eta^{2} \int_{0}^{1} \partial_{x}^{2} u_{\eta} \partial_{x}^{3} \varphi\left(h_{1_{\eta}}\right)=0 .
\end{aligned}
$$

On the one hand, a further integration by parts of the first integral of (35), equation (1), and the energy inequality (21) give

$$
\begin{aligned}
& 4 v_{1} \int_{0}^{1}\left(\partial_{t} u_{\eta}+u_{\eta} \partial_{x} u_{\eta}\right) \partial_{x} h_{1_{\eta}} \\
& =4 v_{1}\left(\frac{d}{d t} \int_{0}^{1} u_{\eta} \partial_{x} h_{1_{\eta}}-\int_{0}^{1} u_{\eta} \partial_{x t}^{2} h_{1_{\eta}}+\int_{0}^{1} u_{\eta} \partial_{x} u_{\eta} \partial_{x} h_{1_{\eta}}\right) \\
& =4 v_{1}\left(\frac{d}{d t} \int_{0}^{1} u_{\eta} \partial_{x} h_{1_{\eta}}-\int_{0}^{1} \partial_{x} u_{\eta} \partial_{x}\left(h_{1_{\eta}} u_{\eta}\right)+\int_{0}^{1} u_{\eta} \partial_{x} u_{\eta} \partial_{x} h_{1_{\eta}}\right) \\
& =4 v_{1}\left(\frac{d}{d t} \int_{0}^{1} u_{\eta} \partial_{x} h_{1_{\eta}}-\int_{0}^{1} h_{1_{\eta}}\left(\partial_{x} u_{\eta}\right)^{2}\right) \\
& =\frac{d}{d t} \int_{0}^{1}\left[4 v_{1} u_{\eta} \partial_{x} h_{1_{\eta}}+\frac{1}{2} h_{1_{\eta}}\left|u_{\eta}\right|^{2}+U\left(h_{1_{\eta}}\right)+\frac{1}{2} g(1-r)\left|h_{1_{\eta}}\right|^{2}+\frac{1}{2} r g\left|h_{1_{\eta}}+h_{2_{\eta}}\right|^{2}+\frac{1}{2} \sigma\left|\partial_{x} h_{1_{\eta}}\right|^{2}\right. \\
& \left.+\frac{\eta}{2}\left|\partial_{x}^{2} h_{1_{\eta}}\right|^{2}+\frac{\eta}{2}\left|\partial_{x}^{3} h_{1_{\eta}}\right|^{2}\right]+\frac{1}{\beta} \int_{0}^{1}\left|u_{\eta}\right|^{2}+r g \varepsilon \int_{0}^{1} \partial_{x} h_{1_{\eta}} \partial_{x} h_{2_{\eta}}+r g \eta \int_{0}^{1} \partial_{x}^{3} h_{1_{\eta}} \partial_{x}^{3} h_{2_{\eta}}+r g \varepsilon \int_{0}^{1}\left|\partial_{x} h_{2_{\eta}}\right|^{2} \\
& +r g^{2} \int_{0}^{1}\left(h_{2_{\eta}}\right)^{2}\left(a+b h_{1_{\eta}}\right)\left(\partial_{x}\left(h_{1_{\eta}}+h_{1_{\eta}}\right)\right)^{2}+4 v_{1} \eta \int_{0}^{1}\left|\partial_{x}^{4} h_{1_{\eta}}\right|^{2}+\eta^{2} \int_{0}^{1} \partial_{x}^{2} u_{\eta} \partial_{x}^{3} \varphi\left(h_{1_{\eta}}\right) \\
& \quad+r g \eta \int_{0}^{1} \partial_{x}^{2} h_{1_{\eta}} \partial_{x}^{2} h_{2_{\eta}}+r g \eta \int_{0}^{1}\left|\partial_{x}^{2} h_{2_{\eta}}\right|^{2} .
\end{aligned}
$$

On the other hand,we can write the third and the fourth integrals of (35) as

$$
\begin{aligned}
&-16 v_{1}^{2} \int_{0}^{1} \partial_{x}\left(\frac{\partial_{x} h_{1_{\eta}}}{h_{1_{\eta}}}\right) \partial_{x} u_{\eta} h_{1_{\eta}}=\frac{1}{2} \frac{d}{d t} \int_{0}^{1} h_{1_{\eta}}\left|\varphi\left(h_{1_{\eta}}\right)\right|^{2}, \\
& \text { - } 4 v_{1} \int_{0}^{1} \frac{u_{\eta} \partial_{x} h_{1_{\eta}}}{\beta h_{1_{\eta}}}=-4 v_{1} \int_{0}^{1} \frac{\partial_{x}\left(u_{\eta} h_{1_{\eta}}\right)}{\beta h_{1_{\eta}}}+4 v_{1} \int_{0}^{1} \frac{\partial_{x} u_{\eta}}{\beta} \\
&=-\frac{1}{\beta} \frac{d}{d t} \int_{0}^{1} \varphi\left(h_{1_{\eta}}\right) .
\end{aligned}
$$

Substituting finally the last three identities into (35), we obtain (22).

\section{Proof of Proposition 1}

We follow the lines performed in (Kitavtsev, Laurençot \& Niethammer, 2011). Using the bound on $\partial_{x} h_{1_{\eta}}$ we obtain:

$$
h_{1_{\eta}}(x, t)-h_{1_{\eta}}(y, t) \leq\left|\int_{x}^{y} \partial_{x} h_{1_{\eta}}(z, t) d z\right| \leq\|x-y\|^{1 / 2}\left\|\partial_{x} h_{1_{\eta}}(t)\right\|_{2} \leq \frac{c_{1}}{\sqrt{\sigma}}|x-y|^{1 / 2}
$$

for all $(x, y) \in(0,1) \times(0,1)$ and $t \in(0, T)$. Next we integrate the above inequality with respect to $y \in(0,1)$, readily give the upper bound. To establish the lower bound for $h_{1_{\eta}}$, we combine the $L^{\infty}\left(0, T ; L^{2}(0,1)\right)$-estimates on $\left(h_{1_{\eta}}\right)^{-3 / 2}$ and $\partial_{x} h_{1_{\eta}}$ just established to obtain a bound on the norm of $1 / \sqrt{h_{1_{\eta}}}$ in $L^{\infty}\left(0, T ; W^{1,1}(0,1)\right)$ since

$$
\int_{0}^{1}\left|\partial_{x}\left(h_{1}^{-1 / 2}\right)\right|=\frac{1}{2} \int_{0}^{1} \frac{\left|\partial_{x} h_{1_{\eta}}\right|}{\left(h_{1_{\eta}}\right)^{3 / 2}} \leq \frac{1}{2 \sqrt{\sigma}}\left\|\sqrt{\sigma} \partial_{x} h_{1_{\eta}}\right\|\left\|_{2}\right\|\left(h_{1_{\eta}}\right)^{-3 / 2} \|_{2} .
$$

Due to the continuous embedding of $W^{1,1}(0,1)$ in $L^{\infty}(0,1)$, we get the positive lower bound.

\section{Proof of Theorem 1}

In this section, we give a proof of the Theorem 1. Let be $\left(h_{1_{\eta_{k}}}, h_{2_{\eta_{k}}}, u_{1_{\eta_{k}}}\right)$ a sequence of weak solutions with initial data

$$
h_{1_{\eta_{k}} \mid t=0}=h_{1_{\eta_{k}}}^{0}, \quad h_{2_{\eta_{k}} \mid t=0}=h_{2_{\eta_{k}}}^{0}, \quad\left(h_{1_{\eta_{k}}} u_{\eta_{k}}\right)_{\mid t=0}=m_{\eta_{k}}^{0}
$$


such as

$$
h_{1_{\eta_{k}}}^{0} \longrightarrow h_{1_{0}} \text { in } H^{1}(\Omega), \quad h_{2_{\eta_{k}}}^{0} \longrightarrow h_{2_{0}} \text { in } H^{1}(\Omega), \quad m_{\eta_{k}}^{0} \longrightarrow m_{0} \text { in }\left(L^{1}(\Omega)\right)^{2},
$$

and satisfying the following inequality:

$$
\begin{aligned}
-\frac{1}{\beta} \int_{0}^{1} \varphi\left(h_{1_{\eta_{k}}}^{0}\right)+\int_{0}^{1}\left[h_{1_{\eta_{k}}}^{0}\left|u_{1_{\eta_{k}}}^{0}\right|^{2}+\right. & 64 v_{1}^{2}\left|\partial_{x} \sqrt{h_{1_{\eta_{k}}}^{0}}\right|^{2}+\frac{1}{2} g(1-r)\left|h_{1_{\eta_{k}}}^{0}\right|^{2}+\frac{1}{2} r g\left|h_{1_{\eta_{k}}}^{0}+h_{2_{\eta_{k}}}^{0}\right|^{2}+\frac{1}{2} \sigma\left|\partial_{x} h_{1_{\eta_{k}}}^{0}\right|^{2} \\
+ & \left.\frac{\eta}{2}\left|\partial_{x}^{3} h_{1_{\eta_{k}}}^{0}\right|^{2}\right] \leq C .
\end{aligned}
$$

Take a sequence $\left\{\eta_{k}\right\}_{k \geq 1} \rightarrow 0$ and, for each $k \geq 1$, denote the corresponding solution to the approximate system (14)-(16)(18)-(19) with $\eta=\eta_{k}$ by $\left(h_{1_{\eta_{k}}}, h_{2_{\eta_{k}}}, u_{\eta_{k}}\right)$.

Convergence of $\sqrt{h_{1_{\eta_{k}}}}, \quad h_{1_{\eta_{k}}}$ and $h_{2_{\eta_{k}}}$

From the remark 6:

$$
\sqrt{h_{\eta_{\eta_{k}}}} \text { is bounded in } L^{\infty}\left(0, T ; H^{1}(\Omega)\right) \text {. }
$$

Moreover, using the mass equation, we obtain the following equality:

$$
\partial_{t} \sqrt{h_{1_{\eta_{k}}}}=\frac{1}{2} \sqrt{h_{1_{\eta_{k}}}} \partial_{x} u_{\eta_{k}}-\partial_{x}\left(\sqrt{h_{1_{\eta_{k}}}} u_{\eta_{k}}\right),
$$

which gives that $\partial_{t} \sqrt{h_{1_{\eta_{k}}}}$ is bounded in $L^{2}\left(0, T ; H^{-1}(\Omega)\right)$.

Applying Aubin-Simon lemma (see (Lions, 1989; Simon, 1987), we can extract a subsequence, still denoted $\left(h_{1_{\eta_{k}}}\right)_{1 \leq k}$, such as

$$
\sqrt{h_{1_{\eta_{k}}}} \text { converges strongly to } \sqrt{h_{1}} \text { in } C^{0}\left(0, T ; L^{2}(0,1)\right) \text {. }
$$

According to the Proposition 1, we show that

$$
\left|h_{1_{\eta_{k}}}-h_{1}\right| \leq \sqrt{c_{2}}\left|\sqrt{h_{1_{\eta_{k}}}}-\sqrt{h_{1}}\right| \Rightarrow\left|h_{1_{\eta_{k}}}-h_{1}\right|^{2} \leq c_{2}\left|\sqrt{h_{1_{\eta_{k}}}}-\sqrt{h_{1}}\right|^{2}
$$

This ensures

$$
h_{1_{\eta_{k}}} \text { converges strongly to } h_{1} \text { in } L^{2}\left(0, T ; L^{2}(0,1)\right) .
$$

We have $h_{2_{\eta_{k}}}$ bounded in $L^{2}\left(0, T ; H^{1}(0,1)\right)$. Moreover, we have

$$
\partial_{t} h_{\eta_{\eta_{k}}}=-\partial_{x}\left(h_{2_{\eta_{k}}} u_{\eta_{k}}\right)+\varepsilon \partial_{x}^{2} h_{2_{\eta_{k}}}+\eta_{k}\left(\partial_{x}^{6} h_{2_{\eta_{k}}}+\partial_{x}^{4} h_{\eta_{\eta_{k}}}\right)+\partial_{x}\left(\left(a h_{\eta_{\eta_{k}}}+b\left(h_{\eta_{\eta_{k}}}\right)^{3}\right) \partial_{x} p_{2_{\eta_{k}}}\right) \text {. }
$$

Let us study each term separately

- Since $h_{2 \eta_{k}}$ is in $L^{\infty}\left(0, T ; L^{2}(0,1)\right)$ and $u_{\eta_{k}}$ is in $L^{2}\left(0, T ; L^{2}(0,1)\right)$, we show that the first term is in $L^{2}\left(0, T ; W^{-1,1}(0,1)\right)$.

- For the second term, since $\partial_{x} h_{2}^{\eta_{k}}$ is in $L^{2}\left(0, T ; L^{2}(0,1)\right)$, we have $\partial_{x}^{2} h_{2}^{\eta_{k}}$ in $L^{2}\left(0, T ; W^{-1,1}(0,1)\right)$.

- For the third one, for any $\psi \in C_{0}^{\infty}((0,1) \times(0, T))$, using integration by parts and regularities in the previous section,

$$
\begin{aligned}
\left|\int_{0}^{T} \int_{0}^{1} \psi \partial_{x}^{6} h_{2 \eta_{k}}\right| & \leq\left\|\partial_{x}^{2} \psi\right\|_{L^{2}\left(0, T ; L^{2}(0,1)\right)}\left\|\partial_{x}^{4} h_{2_{\eta_{k}}}\right\|_{L^{2}\left(0, T ; W^{-1,1}(0,1)\right)} \\
& \leq C\left\|\partial_{x}^{4} h_{2_{\eta_{k}}}\right\|_{L^{2}\left(0, T ; W^{-1,1}(0,1)\right)} . \\
\left|\int_{0}^{T} \int_{0}^{1} \psi \partial_{x}^{4} h_{2 \eta_{k}}\right| & \leq\left\|\partial_{x}^{2} \psi\right\|_{L^{2}\left(0, T ; L^{2}(0,1)\right)}\left\|\partial_{x}^{3} h_{2_{\eta_{k}}}\right\|_{L^{2}\left(0, T ; W^{-1,1}(0,1)\right)} \\
& \leq C\left\|\partial_{x}^{3} h_{2_{\eta_{k}}}\right\|_{L^{2}\left(0, T ; W^{-1,1}(0,1)\right)} .
\end{aligned}
$$

-For the last term, as $h_{2}^{\eta_{k}} \sqrt{a+b h_{2}^{\eta_{k}}}\left(\partial_{x}\left(h_{1_{\eta_{k}}}+h_{2}^{\eta_{k}}\right)\right)$ is in $L^{2}\left(0, T ; L^{2}(0,1)\right)$, we have $\partial_{x}\left(h_{2}^{\eta_{k}} \sqrt{a+b h_{2}^{\eta_{k}}}\left(\partial_{x}\left(h_{1_{\eta_{k}}}+h_{2}^{\eta_{k}}\right)\right)\right)$ in $L^{2}\left(0, T ; W^{-1,1}(0,1)\right)$.

So, the third term is in $L^{2}\left(0, T ; W^{-1,1}(0,1)\right)$ and therefore, $\partial_{t} h_{2_{\eta_{k}}}$ is in $L^{2}\left(0, T ; W^{-1,1}(0,1)\right)$. 
Convergence of $h_{1_{\eta_{k}}} u_{\eta_{k}}$

According to the remark $5, u_{\eta_{k}} \in L^{2}\left(0, T ; H^{1}(0,1)\right)$. This fact with the lemma 1 allows us to get

$$
\left(h_{1_{\eta_{k}}} u_{\eta_{k}}\right) \text { in } L^{2}\left(0, T ; H^{1}(0,1)\right)
$$

Moreover, the momentum equation (2) enables us to write the time derivation of the water discharge:

$$
\begin{aligned}
& \partial_{t}\left(h_{1_{\eta_{k}}} u_{\eta_{k}}\right)=-\partial_{x}\left(h_{1_{\eta_{k}}} u_{\eta_{k}}{ }^{2}\right)-\frac{1}{2} g \partial_{x} h_{\eta_{\eta_{k}}}{ }^{2}+4 v_{1} \partial_{x}\left(h_{1_{\eta_{k}}} \partial_{x} u_{\eta_{k}}\right)-\frac{u_{\eta_{k}}}{\beta}+h_{1_{\eta_{k}}} \partial_{x}\left(\sigma \partial_{x}^{2} h_{\eta_{\eta_{k}}}-V\left(h_{1_{\eta_{k}}}\right)\right) \\
& -r g h_{1_{\eta_{k}}} \partial_{x} h_{2_{\eta_{k}}}-r g h_{\eta_{\eta_{k}}} \partial_{x}\left(h_{1_{\eta_{k}}}+h_{2 \eta_{k}}\right)+\eta_{k} h_{1_{\eta_{k}}}\left(\partial_{x}^{7} h_{1_{\eta_{k}}}+\partial_{x}^{3} h_{1_{\eta_{k}}}\right)-\eta_{k}^{2} \partial_{x}^{4} u_{\eta_{k}} .
\end{aligned}
$$

We then study each term:

- $\partial_{x}\left(h_{1_{\eta_{k}}}\left(u_{\eta_{k}}\right)^{2}\right)=\partial_{x}\left(\left(h_{1_{\eta_{k}}} u_{\eta_{k}}\right) u_{\eta_{k}}\right)$ which is in $L^{2}\left(0, T ; W^{-1,1}(0,1)\right)$.

- As $h_{1_{\eta_{k}}}$ is in $L^{\infty}\left(0, T ; L^{2}(0,1)\right)$, we have:

$$
\partial_{x}\left[\left(h_{1_{\eta_{k}}}\right)^{2}\right] \text { is in } L^{\infty}\left(0, T ; W^{-1,1}(0,1)\right) .
$$

- $\partial_{x}\left(h_{1_{\eta_{k}}} \partial_{x} u_{\eta_{k}}\right)=\partial_{x}\left(\sqrt{h_{\eta_{\eta_{k}}}} \sqrt{h_{\eta_{\eta_{k}}}} \partial_{x} u_{\eta_{k}}\right)$ is bounded in $L^{2}\left(0, T ; W^{-1,1}(0,1)\right)$.

- $r g h_{\eta_{\eta_{k}}} \partial_{x} h_{\eta_{\eta_{k}}}$ is bounded in $L^{2}\left(0, T ; W^{-1,1}(0,1)\right)$.

- $h_{1_{\eta_{k}}} \partial_{x} \partial_{x}^{2} h_{1_{\eta_{k}}}$ is bounded in $L^{\infty}\left(0, T ; W^{-1,1}(0,1)\right)$.

- $r g h_{\eta_{\eta_{k}}} \partial_{x}\left(h_{1_{\eta_{k}}}+h_{2_{\eta_{k}}}\right)$ is bounded in $L^{2}\left(0, T ; W^{-1,1}(0,1)\right)$.

- For any $\psi \in C_{0}^{\infty}((0, T) \times(0,1))$, we obtain, using integration by parts and the regularities in the previous section,

$$
\begin{aligned}
& \left|\int_{0}^{T} \int_{0}^{1} \psi h_{1_{\eta_{k}}} \partial_{x}^{7} h_{1_{\eta_{k}}}\right|=\left|\int_{0}^{T} \int_{0}^{1} \partial_{x}^{4} h_{1_{\eta_{k}}}\left[\psi \partial_{x}^{3} h_{1_{\eta_{k}}}+3 \partial_{x} \psi \partial_{x}^{2} h_{1_{\eta_{k}}}+3 \partial_{x}^{2} \psi \partial_{x} h_{1_{\eta_{k}}}+h_{1_{\eta_{k}}} \partial_{x}^{3} \psi\right]\right| \\
& \leq \int_{0}^{T}\left\|\partial_{x}^{4} h_{1_{\eta_{k}}}\right\|_{L^{2}(0,1)}\left[\|\psi\|_{L^{\infty}(0,1)}\left\|\partial_{x}^{3} h_{1_{\eta_{k}}}\right\|_{L^{2}(0,1)}+\left\|h_{1_{\eta_{k}}}\right\|_{L^{\infty}(0,1)}\left\|\partial_{x}^{3} \psi\right\|_{L^{2}(0,1)}\right. \\
& \left.+3\left\|\partial_{x} \psi\right\|_{L^{\infty}(0,1)}\left\|\partial_{x}^{2} h_{1_{\eta_{k}}}\right\|_{L^{2}(0,1)}+3\left\|\partial_{x}^{2} \psi\right\|_{L^{\infty}}\left\|\partial_{x} h_{\eta_{\eta_{k}}}\right\|_{L^{2}(0,1)}\right] \\
& \leq C\left\|\partial_{x}^{4} h_{1_{\eta_{k}}}\right\|_{L^{2}(0,1)}^{2}\|\psi\|_{H^{3}(0,1)} \leq\|\psi\|_{L^{2}\left(0, T ; H^{3}(0,1)\right)}, \\
& \left|\int_{0}^{T} \int_{0}^{1} \psi h_{1_{\eta_{k}}} \partial_{x} V\left(h_{\eta_{\eta_{k}}}\right)\right|=\left|\int_{0}^{T} \int_{0}^{1} \partial_{x} \psi V_{1}\left(h_{1_{\eta_{k}}}\right)\right| \\
& \leq\left\|V_{1}\left(h_{1_{\eta_{k}}}\right)\right\|_{L^{\infty}((0,1) \times(0, T))}\left(\int_{0}^{T}\|\psi\|_{H^{1}(0,1)}\right)^{\frac{1}{2}}
\end{aligned}
$$

where $V_{1}\left(h_{1_{\eta_{k}}}\right):=-\int_{h}^{\infty} \tau V_{1}^{\prime}(\tau) d \tau$,

and

$$
\begin{gathered}
\left|\int_{0}^{T} \int_{0}^{1} \psi h_{1_{\eta_{k}}} \partial_{x}^{3} h_{1_{\eta_{k}}} d x d t\right|=\left|\int_{0}^{T} \int_{0}^{1} \partial_{x}^{2} h_{1_{\eta_{k}}}\left[h_{1_{\eta_{k}}} \partial_{x} \psi+\psi \partial h_{1_{\eta_{k}}}\right]\right| \\
\leq \int_{0}^{T}\left\|\partial_{x}^{2} h_{1_{\eta_{k}}}\right\|_{L^{2}(0,1)}\left[\left\|h_{1_{\eta_{k}}}\right\| L_{L^{\infty}(0,1)}\left\|\partial_{x} \psi\right\|_{L^{2}(0,1)}+\|\psi\|_{L^{\infty}(0,1)}\left\|\partial_{x} h_{1_{\eta_{k}}}\right\|_{L^{2}(0,1)}\right] \\
\leq C\left(\int_{0}^{T}\|\psi\|_{H^{1}(0,1)}^{2}\right)^{\frac{1}{2}} .
\end{gathered}
$$

Finally $\left(u_{\eta_{k}}\right)$ and $\left(\eta_{k} \partial_{x}^{4} u_{\eta_{k}}\right)$ are bounded in $L^{2}\left(0,1 ; H^{1}(0, T)\right)$ and $L^{2}\left(0, T ; H^{-2}(0,1)\right)$ respectively. Collecting the above information completes the proof of the boundness of the right-hand side of (39), whence

$$
\partial_{t}\left(h_{1_{\eta_{k}}} u_{\eta_{k}}\right) \text { is bounded in } L^{2}\left(0, T ; H^{-3}(0,1)\right) \text {. }
$$


Combining this with (38) and corollary 4 in (Simon, 1987) ensures that $\left(h_{1_{\eta_{k}}} u_{\eta_{k}}\right)$ is compact in $L^{2}\left((0, T) ; L^{2}(0,1)\right)$. So, there exists $\mathbf{m} \in L^{2}\left((0, T) ; L^{2}(0,1)\right)$ such that

$$
h_{1_{\eta_{k}}} u_{\eta_{k}} \quad \text { converges to } \mathbf{m} \text { in } \quad L^{2}\left((0, T) ; L^{2}(0,1)\right) \text {. }
$$

Convergences of $\left(h_{1_{\eta_{k}}}\right)^{-1}, u_{\eta_{k}}$ and $\sqrt{h_{\eta_{\eta_{k}}}} u_{\eta_{k}}$

- As $\left(h_{1_{\eta_{k}}}\right)_{k}$ converges strongly to $h_{1}$ in $L^{2}\left(0, T ; W^{1, p}(0,1)\right) \cap C\left([0, T] \times(0,1)\right.$ for $p \in[1, \infty)$ and we have $0<c_{1} \leq h_{1_{\eta_{k}}} \leq c_{2}$, we deduce that

$$
\left(h_{1_{\eta_{k}}}\right)^{-1} \quad \text { converges strongly to } h_{1}^{-1} \text { in } C([0, T] \times(0,1)) .
$$

- Considering (40) and (41), there exists $u_{1} \in L^{2}\left(0, T ; H^{1}(0,1)\right)$ such that

$$
u_{\eta_{k}} \text { converges strongly to } u \text { in } L^{2}\left(0, T ; L^{2}(0,1)\right) \text {. }
$$

- Since $\sqrt{h_{\eta_{\eta_{k}}}}$ converges strongly to $\sqrt{h_{1}}$ in $C^{0}\left(0, T ; L^{2}(0,1)\right)$, by using $(42), \sqrt{h_{\eta_{\eta_{k}}}} u_{\eta_{k}}$ converges strongly to $\sqrt{h_{1}} u_{1}$ in $L^{2}\left(0, T ; L^{1}(0,1)\right)$.

Convergences of $\partial_{x} h_{1_{\eta_{k}}}, \quad h_{2_{\eta_{k}}} \partial_{x} h_{1_{\eta_{k}}}, \quad \partial_{x}^{2} h_{1_{\eta_{k}}}, \quad h_{1_{\eta_{k}}} \partial_{x}^{2} h_{1_{\eta}}$ and $\partial_{x} h_{1_{\eta_{k}}} \partial_{x}^{2} h_{1_{\eta_{k}}}$

- We have $\partial_{x} h_{1_{\eta_{k}}}$ bounded in $L^{2}\left(0, T ; H^{1}(0,1)\right)$ and $\partial_{t} \partial_{x} h_{1_{\eta_{k}}}$ is bounded in $L^{\infty}\left(0, T ; H^{-1}(0,1)\right)$ since $\partial_{t} h_{1_{\eta_{k}}}$ is bounded in $L^{\infty}\left(0, T ; H^{-1}(0,1)\right)$. Thanks to compact injection of $H^{1}(0,1)$ in $L^{2}(0,1)$ in one dimension, we have:

$$
\partial_{x} h_{1_{\eta_{k}}} \quad \text { converges strongly to } \partial_{x} h_{1} \quad \text { in } L^{2}\left(0, T ; L^{2}(0,1)\right) .
$$

- The bound of $\partial_{x}^{2} h_{1_{\eta_{k}}}$ in $L^{2}\left(0, T ; L^{2}(0,1)\right)$ and $\partial_{x} h_{2_{\eta_{k}}}$ in $L^{2}\left(0, T ; L^{2}(0,1)\right)$ gives us:

$$
\begin{aligned}
& \partial_{x}^{2} h_{1_{\eta_{k}}} \quad \text { converges strongly to } \quad \partial_{x}^{2} h_{1} \quad \text { in } L^{1}\left(0, T ; L^{1}(0,1)\right) \text {, } \\
& \partial_{x} h_{2 \eta_{k}} \quad \text { converges strongly to } \quad \partial_{x} h_{2} \quad \text { in } L^{1}\left(0, T ; L^{1}(0,1)\right) .
\end{aligned}
$$

- Thanks to the strong convergence of $h_{1_{\eta_{k}}}, h_{2_{\eta_{k}}}, \partial_{x} h_{1_{\eta_{k}}}$ and the weak convergence of $\partial_{x}^{2} h_{\eta_{\eta_{k}}}$, we have:

$$
\begin{array}{rll}
h_{2_{\eta_{k}}} \partial_{x} h_{\eta_{\eta_{k}}} & \text { converges strongly to } & h_{2} \partial_{x} h_{1} \quad \text { in } L^{1}\left(0, T ; L^{1}(0,1)\right), \\
h_{\eta_{\eta_{k}}} \partial_{x}^{2} h_{1_{\eta_{k}}} & \text { converges strongly to } & h_{1} \partial_{x}^{2} h_{1} \quad \text { in } L^{1}\left(0, T ; L^{1}(0,1)\right), \\
\partial_{x} h_{1_{\eta_{k}}} \partial_{x}^{2} h_{\eta_{\eta_{k}}} & \text { converges wealkly to } & \partial_{x} h_{1} \partial_{x}^{2} h_{1} \quad \text { in } L^{1}\left(0, T ; L^{1}(0,1)\right), \\
h_{1_{\eta_{k}}} \partial_{x} h_{2_{\eta_{k}}} & \text { converges strongly to } & h_{1} \partial_{x} h_{2} \quad \text { in } L^{1}\left(0, T ; L^{1}(0,1)\right), \\
h_{2_{\eta_{k}}} \partial_{x} h_{\eta_{\eta_{k}}} & \text { converges strongly to } & h_{2} \partial_{x} h_{2} \quad \text { in } L^{1}\left(0, T ; L^{1}(0,1)\right), \\
\left(h_{1_{\eta_{k}}}\right)^{2} & \text { converges strongly to } & h_{1}^{2} \quad \text { in } L^{1}\left(0, T ; L^{1}(0,1)\right), \\
\left(h_{\eta_{\eta_{k}}}\right)^{2} & \text { converges strongly to } & h_{2}^{2} \quad \text { in } L^{1}\left(0, T ; L^{1}(0,1)\right), \\
h_{\eta_{\eta_{k}}} h_{\eta_{\eta_{k}}} & \text { converges strongly to } & h_{1} h_{2} \quad \text { in } L^{1}\left(0, T ; L^{1}(0,1)\right) .
\end{array}
$$

Convergences of $h_{1_{\eta_{k}}} \partial_{x} u_{\eta_{k}}, u_{\eta_{k}}$

As $u_{\eta_{k}}$ is bounded in $L^{2}\left(0, T ; L^{2}(0,1)\right)$, then $\partial_{x} u_{\eta_{k}}$ is bounded in $L^{2}\left(0, T ; W^{-1,2}(0,1)\right)$.

Then,

$$
u_{\eta_{k}} \quad \text { converges strongly to } \quad u \quad \text { in } L^{1}\left(0, T ; L^{1}(0,1)\right) \text {. }
$$

At last, the function $\left(h_{1_{\eta_{k}}}, \partial_{x} u_{\eta_{k}}\right) \longmapsto h_{1_{\eta_{k}}} \partial_{x} u_{\eta_{k}}$ is a continuous in $L^{\infty}\left(0, T ; H^{1}(0,1)\right) \times L^{2}\left(0, T ; W^{-1,2}(0,1)\right)$ to $L^{2}\left(0, T ; W^{-1,2}(0,1)\right)$. So,

$$
h_{1_{\eta_{k}}} \partial_{x} u_{\eta_{k}} \quad \text { converges weakly to } \quad h_{1} \partial_{x} u \text { in } L^{2}\left(0, T ; H^{-1}(0,1)\right) \text {. }
$$


Convergences of $h_{2_{\eta_{k}}} u_{\eta_{k}}$ and $\partial_{x}^{2} h_{\eta_{\eta_{k}}}$

We know that $\partial_{x} h_{2_{\eta_{k}}}$ is bounded in $L^{2}\left(0, T ; L^{2}(0,1)\right)$ this implies $\partial_{x}^{2} h_{2}^{k}$ is in $L^{1}\left(0, T ; W^{-1,2}(0,1)\right)$.

So,

$$
\partial_{x}^{2} h_{2 \eta_{k}} \quad \text { converges weakly to } \quad \partial_{x}^{2} h_{2} \in L^{1}\left(0, T ; W^{-1,2}(0,1)\right) .
$$

To conclude, we have $u_{\eta_{k}}$ converges weakly to $u$ in $L^{2}\left(0, T ; L^{2}(0,1)\right)$ and the strong convergence of $h_{2}^{k}$ to $h_{2}$, gives us:

$$
h_{\eta_{\eta_{k}}} u_{\eta_{k}} \quad \text { converges weakly to } h_{2} u \quad \text { in } L^{1}\left(0, T ; L^{1}(0,1)\right) .
$$

Convergence of $\left.\left(a\left(h_{2_{\eta_{k}}}\right)^{2}+b\left(h_{2_{\eta_{k}}}\right)^{3}\right)\right) \partial_{x}\left(h_{1_{\eta_{k}}}+h_{2_{\eta_{k}}}\right)$

We know that $\partial_{x}\left(h_{1_{\eta_{k}}}+h_{2_{\eta_{k}}}\right)$ converges weakly to $\partial_{x}\left(h_{1}+h_{2}\right)$ in $L^{2}\left(0, T ; L^{2}(0,1)\right)$ and $\left(a\left(h_{2_{\eta_{k}}}\right)^{2}+b\left(h_{2_{\eta_{k}}}\right)^{3}\right)$ converges strongly to $a h_{2}^{2}+b h_{2}^{3}$ in $L^{1}\left(0, T ; L^{1}(0,1)\right)$.

So,

$$
\left.\left(a\left(h_{\eta_{\eta_{k}}}\right)^{2}+b\left(h_{\eta_{\eta_{k}}}\right)^{3}\right)\right) \partial_{x}\left(h_{1_{\eta_{k}}}+h_{1_{\eta_{k}}}\right) \quad \text { converges weakly to } \quad\left(a h_{2}^{2}+b h_{2}^{3}\right) \partial_{x}\left(h_{1}+h_{2}\right) \quad \text { in } L^{1}\left(0, T ; L^{1}(0,1)\right)
$$

Convergences of $h_{1_{\eta}} V\left(h_{1_{\eta_{k}}}\right)$ and $V\left(h_{1_{\eta_{k}}}\right) \partial_{x} h_{1_{\eta_{k}}}$

We will begin by studying the convergence of the term $h_{1} V\left(h_{1_{\eta_{k}}}\right)$. We have $h_{1} V\left(h_{1_{\eta_{k}}}\right)=\frac{1}{\left(h_{\eta_{\eta_{k}}}\right)^{2}}-\frac{\alpha}{\left(h_{\eta_{\eta_{k}}}\right)^{3}}$ and

$$
\begin{gathered}
\left|\frac{1}{\left(h_{\eta_{\eta_{k}}}\right)^{2}}-\frac{\alpha}{\left(h_{\eta_{\eta_{k}}}\right)^{3}}-\left(\frac{1}{h_{1_{\eta}}^{2}}-\frac{\alpha}{h_{1_{\eta}}^{3}}\right)\right| \leq\left|\frac{1}{\left(h_{\eta_{\eta_{k}}}\right)^{2}}-\frac{1}{h_{1}^{2}}\right|+\left|\frac{1}{\left(h_{1_{\eta_{k}}}\right)^{3}}-\frac{1}{h_{1}^{3}}\right| \\
\left|\frac{1}{\left(h_{1_{\eta_{k}}}\right)^{2}}-\frac{\alpha}{\left(h_{\eta_{\eta_{k}}}\right)^{3}}-\left(\frac{1}{h_{1}^{2}}-\frac{\alpha}{h_{1}^{3}}\right)\right| \leq \frac{\left|h_{\eta_{\eta_{k}}}-h_{1}\right|\left|h_{1_{\eta_{k}}}+h_{1}\right|}{\left(h_{1_{\eta_{k}}}\right)^{2} h_{1}^{2}}+\frac{\left|h_{1_{\eta_{k}}}-h_{1} \|\left(h_{1_{\eta_{\eta_{k}}}}\right)^{2}+h_{1_{\eta_{k}}} h_{1}+h_{1}^{2}\right|}{\left(h_{\eta_{\eta_{k}}}\right)^{3} h_{1}^{3}} .
\end{gathered}
$$

We use the Proposition 1 to find two constants $\delta_{1}$ and $\delta_{2}$ such as

$$
\left|\frac{1}{\left(h_{\eta_{\eta_{k}}}\right)^{2}}-\frac{\alpha}{\left(h_{\eta_{\eta_{k}}}\right)^{3}}-\left(\frac{1}{h_{1}^{2}}-\frac{\alpha}{h_{1}^{3}}\right)\right| \leq \delta_{1}\left|h_{1_{\eta_{k}}}-h_{1}\right|+\delta_{2}\left|h_{1_{\eta_{k}}}-h_{1}\right| \text {. }
$$

So

$$
\left|\frac{1}{\left(h_{\eta_{\eta_{k}}}\right)^{2}}-\frac{\alpha}{\left(h_{\eta_{\eta_{k}}}\right)^{3}}-\left(\frac{1}{h_{1}^{2}}-\frac{\alpha}{h_{1}^{3}}\right)\right|^{2} \leq \delta_{3}^{2}\left|h_{1}^{k}-h_{1}\right|^{2} \rightarrow 0, \quad \text { with } \delta_{3}=2 \max \left(\delta_{1}, \delta_{2}\right) \text {. }
$$

We have

$$
\frac{1}{\left(h_{\eta_{\eta_{k}}}\right)^{2}}-\frac{\alpha}{\left(h_{\eta_{\eta_{k}}}\right)^{3}} \quad \text { converges strongly to } \quad \frac{1}{h_{1}^{2}}-\frac{\alpha}{h_{1}^{3}} \text { in } L^{2}\left(0, T ; L^{2}(0,1)\right) \text {. }
$$

A similar reasoning ensures the strong convergence of $\frac{1}{\left(h_{1_{\eta_{k}}}\right)^{3}}-\frac{\alpha}{\left(h_{\eta_{\eta_{k}}}\right)^{4}} \quad$ to $\frac{1}{h_{1}^{3}}-\frac{\alpha}{h_{1}^{4}}$ in $L^{2}\left(0, T ; L^{2}(0,1)\right)$.

The strong convergence of $\partial_{x} h_{1_{\eta_{k}}}$ in $L^{2}\left(0, T ; L^{2}(0,1)\right)$ gives us

$$
V\left(h_{1_{\eta_{k}}}\right) \partial_{x} h_{\eta_{\eta_{k}}} \quad \text { converges weakly to } \quad V\left(h_{1}\right) \partial_{x} h_{1} \quad \text { in } L^{1}\left(0, T ; L^{1}(0,1)\right) .
$$

Convergence of $\partial_{x}^{2} u_{\eta_{k}}, h_{1_{\eta_{k}}} \partial_{x}^{7} h_{1_{\eta_{k}}}, \partial_{x}^{6} h_{\eta_{\eta_{k}}}$

- The bound of $\partial_{x}^{2} u_{\eta_{k}}$ in $L^{2}\left(0, T ; L^{2}(0,1)\right)$ gives us:

$$
\partial_{x}^{2} u_{\eta_{k}} \quad \text { converges strongly to } \quad \partial_{x}^{4} u \quad \text { in } L^{1}\left(0, T ; L^{1}(0,1)\right) .
$$

-, we have:

$$
\begin{aligned}
& \int_{0}^{T} \int_{0}^{1} h_{1_{\eta_{k}}} \partial_{x}^{7} h_{1_{\eta_{k}}} \phi=-3 \int_{0}^{T} \int_{0}^{1} \partial_{x}^{2} h_{\eta_{\eta_{k}}} \partial x \phi \partial_{x}^{4} h_{1_{\eta_{k}}}-3 \int_{0}^{T} \int_{0}^{1} \partial_{x} h_{1_{\eta_{k}}} \partial_{x}^{2} \phi \partial_{x}^{4} h_{\eta_{\eta_{k}}}-\int_{0}^{T} \int_{0}^{1} \phi \partial_{x}^{3} h_{\eta_{\eta_{k}}} \partial_{x}^{4} h_{1_{\eta_{k}}} \\
& -\int_{0}^{T} \int_{0}^{1} h_{1_{\eta_{k}}} \partial_{x}^{3} \phi \partial_{x}^{4} h_{1_{\eta_{k}}}
\end{aligned}
$$


The function $\left(\partial_{x}^{2} h_{1_{\eta_{k}}}, \partial_{x}^{4} h_{1_{\eta_{k}}}\right) \longmapsto \partial_{x}^{2} h_{1_{\eta_{k}}} \partial_{x}^{4} h_{1_{\eta_{k}}}$ is a continuous in $L^{\infty}\left(0, T ; H^{1}(0,1)\right) \times L^{2}\left(0, T ; W^{-1,2}(0,1)\right)$ to $L^{2}\left(0, T ; W^{-1,2}(0,1)\right)$, so $\left(\partial_{x}^{2} h_{\eta_{\eta_{k}}} \partial_{x}^{4} h_{1_{\eta_{k}}}\right) k$ converges weakly to $\partial_{x}^{2} h_{\eta_{\eta}} \partial_{x}^{4} h_{\eta_{\eta}}$ in $L^{2}\left(0, T ; W^{-1,2}(0,1)\right)$. Next, the function $\left(\partial_{x}^{3} h_{1_{\eta_{k}}}, \partial_{x}^{4} h_{1_{\eta_{k}}}\right) \longmapsto \partial_{x}^{3} h_{1_{\eta_{k}}} \partial_{x}^{4} h_{\eta_{\eta_{k}}}$ is a continuous in $L^{\infty}\left(0, T ; H^{1}(0,1)\right) \times L^{2}\left(0, T ; W^{-1,2}(0,1)\right)$ to $L^{2}\left(0, T ; W^{-1,2}(0,1)\right)$, so $\left(\partial_{x}^{3} h_{1_{\eta_{k}}} \partial_{x}^{4} h_{1_{\eta_{k}}}\right)_{k}$ converges weakly to $\partial_{x}^{3} h_{1} \partial_{x}^{4} h_{1}$ in $L^{2}\left(0, T ; W^{-1,2}(0,1)\right)$.

$\partial_{x} h_{\eta_{\eta_{k}}} \partial_{x}^{4} h_{1_{\eta_{k}}}$ converges weakly to $\partial_{x} h_{1} \partial_{x}^{4} h_{1}$ in $L^{2}\left(0, T ; L^{1}(0,1)\right)$. Finally, $h_{1_{\eta_{k}}} \partial_{x}^{4} h_{\eta_{\eta_{k}}}$ converges strongly to $h_{1} \partial_{x}^{4} h_{1}$ in $L^{1}\left(0, T ; L^{1}(0,1)\right)$.

- We have:

$\int_{0}^{T} \int_{0}^{1} \partial_{x}^{6} h_{\eta_{\eta_{k}}} \phi=\int_{0}^{T} \int_{0}^{1} h_{2_{\eta_{k}}} \partial_{x}^{6} \phi$

The bound of $h_{2 \eta_{k}}$ in $L^{\infty}\left(0, T ; L^{2}(0,1)\right)$, give us:

the strong convergence of $h_{2_{\eta_{k}}}$ in $L^{2}\left(0, T ; W^{-1,1}(0,1)\right)$.

Convergence of $h_{1_{\eta_{k}}} \partial_{x}^{3} h_{1_{\eta_{k}}}$

The function $\left(h_{1_{\eta_{k}}}, \partial_{x}^{3} h_{1_{\eta_{k}}}\right) \longmapsto h_{1_{\eta_{k}}} \partial_{x}^{3} h_{1_{\eta_{k}}}$ is a continuous in $L^{\infty}\left(0, T ; H^{1}(0,1)\right) \times L^{2}\left(0, T ; W^{-1,2}(0,1)\right)$ to $L^{2}\left(0, T ; W^{-1,2}(0,1)\right)$, so $h_{1_{\eta_{k}}} \partial_{x}^{3} h_{1_{\eta_{k}}}$ converges weakly to $h_{1} \partial_{x}^{3} h_{1}$ in $L^{2}\left(0, T ; W^{-1,2}(0,1)\right)$.

These above convergences then allow us to pass to the limit as $n \rightarrow \infty$ in the weak formulation of the approximating systems $(14)-(16)-(18)-(19)$ in order to get that $\left(h_{1}, h_{2}, u_{1}\right)$ satisfies $(24)-(25)$.

\section{Copyrights}

Copyright for this article is retained by the author(s), with first publication rights granted to the journal.

This is an open-access article distributed under the terms and conditions of the Creative Commons Attribution license (http://creativecommons.org/licenses/by/4.0/). 